\title{
Anisotropies in the Hı gas distribution toward 3C 196
}

\author{
P. M. W. Kalberla and J. Kerp
}

\begin{abstract}
Argelander-Institut für Astronomie, Universität Bonn, Auf dem Hügel 71, 53121 Bonn, Germany e-mail: pkalberla@astro.uni-bonn.de
\end{abstract}

Received 14 June 2016 / Accepted 18 August 2016

\begin{abstract}
Context. The local Galactic Hi gas was found to contain cold neutral medium (CNM) filaments that are aligned with polarized dust emission. These filaments appear to be dominated by the magnetic field and in this case turbulence is expected to show distinct anisotropies.

Aims. We use the Galactic Effelsberg-Bonn HI Survey (EBHIS) to derive 2D turbulence spectra for the HI distribution in direction to 3C 196 and two more comparison fields.

Methods. Prior to Fourier transform we apply a rotational symmetric 50\% Tukey window to apodize the data. We derive average as well as position angle dependent power spectra. Anisotropies in the power distribution are defined as the ratio of the spectral power in orthogonal directions.

Results. We find strong anisotropies. For a narrow range in position angle, in direction perpendicular to the filaments and the magnetic field, the spectral power is on average more than an order of magnitude larger than parallel. In the most extreme case the anisotropy reaches locally a factor of 130 . Anisotropies increase on average with spatial frequency as predicted by Goldreich \& Sridhar (1995, ApJ, 438, 763), at the same time the Kolmogorov spectral index remains almost unchanged. The strongest anisotropies are observable for a narrow range in velocity and decay with a power law index close to $-8 / 3$, almost identical to the average isotropic spectral index of $-2.9<\gamma<-2.6$.

Conclusions. HI filaments, associated with linear polarization structures in LOFAR observations in direction to 3C 196, show turbulence spectra with marked anisotropies. Decaying anisotropies appear to indicate that we witness an ongoing shock passing the HI and affecting the observed Faraday depth.
\end{abstract}

Key words. turbulence - ISM: structure - ISM: magnetic fields

\section{Introduction}

The basic theory of a turbulent interstellar medium (ISM) with the concept of the hierarchy of eddies was outlined by von Weizsäcker (1951). The energy flow from large eddies to smaller ones was described in terms of wave numbers $k$, the reciprocal length of the associated Fourier component, giving rise to the well known Kolmogorov relation for the power distribution $P(k) \propto k^{-5 / 3}$. In a more general way for the decay of turbulent energy within a compressible medium a constant power law index $P(k) \propto k^{-\gamma}$ is expected. When the turbulent flow reaches the dissipative range where turbulent motion is converted to heat, the power distribution should steepen strongly, $P(k) \propto k^{-7}$.

The measurement of $P(k)$ is, in principle, straightforward for radio synthesis telescopes by using the squares of the observed visibility amplitudes at various baselines. In practice, however, a number of important details such as calibration, noise performance, UV plane coverage (sampling bias) and projection effects need to be taken into account (Green 1993). Most important is to apply a uniform taper in the UV plane which is usually avoided when generating sensitive interferometer maps (Briggs 1995). Inappropriate tapering may degrade power spectra seriously.

Single-dish radio telescopes can be used to generate 1D power spectra from drift scans or average power spectra from 2D maps. Tapering issues, discussed later in more detail (Sect. 3.2), are also in this case important. Essentially 2D single-dish data have to be processed analogous to interferometer data for an accurate power spectrum analysis; instrumental effects need to be eliminated carefully.

Attempts to quantify ISM power law indices for a turbulent HI distribution were made by several authors. In the first instance 2D interferometer channel maps, slices of the observed brightness temperature distribution at a constant velocity, were used. Crovisier \& Dickey (1983) found power law spectra that were independent of position angle with typical values of $\gamma \sim-3$ for Westerbork synthesis telescope observations. From additional 1D Arecibo and Nançay drift scans they derived $\gamma \sim-2$. Erroneously it was concluded that interferometer and single-dish telescopes sample HI distributions with different properties. This issue was solved later, as it was realized that power laws derived from $1 \mathrm{D}$ and 2D distributions result in power law indices differing by one (Green 1993).

Kalberla \& Mebold (1983) used also Westerkork observations, supplemented by short spacing data from the Effelsberg 100-m telescope, and derived for local HI gas toward 3C 147 $\gamma=-2.5 \pm 0.3$. Using the DRAO Galactic Plane Survey for HI emission, Green (1993) derived power law indices between $-3<\gamma<-2.2$. More distant HI gas, which corresponds to large physical scales, tends to have more negative indices than the nearer HI .

After these early investigations it became obvious that turbulence in the HI gas cannot be described by a unique power law. Rather turbulence in the $\mathrm{HI}$ is governed by processes that inject the kinetic energy to the ISM with different decay processes. In fact, the power spectrum of a velocity channel 
is a complex mixture of velocity and density fluctuations. Lazarian \& Pogosyan (2000) studied these issues in detail. They argued that intensity fluctuations within individual channel maps are generated predominantly by turbulent velocity fields. Increasing the thickness of the observed velocity slices causes density fluctuations to dominate the emissivity. For thin slices in velocity preferentially a power law index close to $\gamma=-8 / 3$, the 2D Kolmogorov index, is expected. The analysis of column density maps (thick slices in velocity) should result in power law indices that are steeper by one (Lazarian \& Pogosyan 2000, Eq. (28)). Esquivel \& Lazarian (2005) argued that velocity centroids are most useful as tracers of turbulence and favor centroid maps for anisotropy studies.

Using data from the Southern Galactic Plane Survey in the fourth Galactic quadrant, Dickey et al. (2001) derived turbulence spectra with power law indices between $-4<\gamma<-3$, confirming essentially the predicted steepening for column density maps. Miville-Deschênes et al. (2003) studied the Ursa Major Galactic cirrus and found that the spectral index is similar for the 3D density and velocity fields with a value of $-3.6 \pm 0.2$. Also Miville-Deschênes \& Martin (2007) found that the power spectra of the integrated emission and velocity centroids are both well fit by a slope of $-3.4 \pm 0.2$. Using Arecibo data Dedes \& Kalberla (2012) report for thin slices a spectral index of -2.67 for the local gas and -2.5 for the extra-planar component. Martin et al. (2015), using the Green Bank Telescope (GBT), studied the HI distribution in toward the northern ecliptic pole. They derived power spectra of column density maps for low-, intermediate-, and high-velocity gas components (LVC, IVC, and HVC) with indices of $-2.86 \pm 0.04,-2.69 \pm 0.04$, and $-2.59 \pm 0.07$, respectively. Blagrave et al. (2016) observed several fields at intermediate Galactic latitudes with the DRAO and the GBT and find exponents ranging from -2.5 to -3.0 . Power spectra of maps of the centroid velocity for these components give similar results.

For the Small Magellanic Cloud (SMC) Stanimirovic et al (1999) derived power law indices in the range $\gamma=-3.04 \pm 0.02$. This implies that the dynamics of the HI distribution are similar for both the Galaxy and the SMC.

All these investigation are consistent with the assumption that the ISM is dominated by turbulence. Since a few years increasingly attention was drawn to the fact that typical astrophysical fluids are accompanied by magnetic fields. These fields may be strong enough to affect the dynamics of the turbulent cascade from large to small eddies. The motion along the magnetic field is free and unaffected, but perpendicular to the field motions are hindered. During the energy cascade this leads to increasingly asymmetric eddies. Thus magneto-hydrodynamical (MHD) turbulence in presence of a magnetic field is expected to be strongly anisotropic (Goldreich \& Sridhar 1995), for reviews see Cho et al. (2003), Brandenburg \& Lazarian (2013).

So far, imprints to the spectral power distribution from anisotropies in the HI distribution were not reported. However recently new powerful single-dish HI surveys became available. For the northern sky there is the Galactic Effelsberg-Bonn HI Survey (EBHIS, Winkel et al. 2016a), in the south the Parkes Galactic All Sky Survey (GASS, McClure-Griffiths et al. 2009; Kalberla et al. 2010). Kalberla et al. (2016), merging EBHIS with the 3rd release of the GASS (GASS III, Kalberla \& Haud 2015), have shown that the cold neutral medium (CNM) in the local vicinity is mostly organized in filaments. A comparison with structures measured by Planck at $353 \mathrm{GHz}$ (Planck Collaboration XXXII 2016) disclosed that the CNM filaments are well aligned with the thermal dust emission.

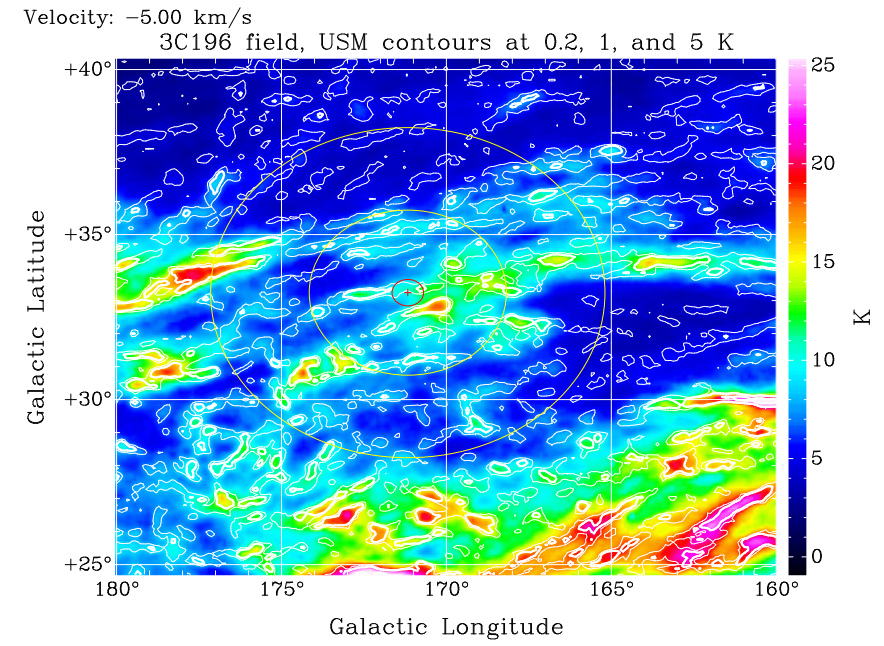

Fig. 1. Brightness temperature distribution in direction to 3C 196 in Galactic coordinates at a velocity of $-5 \mathrm{~km} \mathrm{~s}^{-1}$ on large scales. 3C 196 is marked in red, the yellow circles have radii of $2.5^{\circ}$ and $5^{\circ}$. Filamentary features from USM maps are overlaid with contours of 0.2 , 1 , and $5 \mathrm{~K}$.

Moreover, the HI filaments are well aligned with the magnetic field direction measured by Planck and anisotropies in the CNM distribution are common. We therefore find that a search for anisotropic MHD turbulence is overdue and decided to dedicate this analysis to anisotropies in HI turbulence spectra.

\section{Source selection}

Linear polarization structures in LOFAR observations of the ISM in the 3C 196 field have been observed by Jelić et al. (2015). This is one of the three primary fields of the LOFAR-Epoch of Reionization key science project. The high band antennas (HBA) have been used to image this region and the distribution of polarized structures in Faraday depth was derived by rotation measure (RM) synthesis.

These observations disclosed an interesting morphological feature, a strikingly straight filament at a Faraday depth of $+0.5 \mathrm{rad} \mathrm{m}^{-2}$, approximately in north/south direction in the center across 3C 196, and parallel to the Galactic plane. The width of the central filament is roughly 8 to $10 \mathrm{arcmin}$. Moreover, there are also linear depolarization canals conspicuous in images at the peaks of the Faraday spectra. Jelić et al. (2015) conclude that the filamentary structure is probably caused by an ionized filament in the ISM, located somewhere in the foreground.

We used the EBHIS to study the HI distribution in direction to 3C 196. The LOFAR field field is located in a region at intermediate Galactic latitudes that is dominated by numerous HI filaments, running approximately parallel to the Galactic plane. The large scale structure of the filaments is best visualized at a velocity of $-5 \mathrm{~km} \mathrm{~s}^{-1}$. Figure 1 displays color coded an overview of the observed brightness temperature $T_{\mathrm{B}}$. Filaments are best visible after unsharp masking (USM) the observations. We generated USM maps by subtracting from the observed $T_{\mathrm{B}}$ distribution a smoothed distribution with an effective resolution of 0.5 (see Kalberla et al. 2016, for details). For the local gas the resulting filamentary structures are found to have low Doppler temperatures, characteristic for the CNM. This gas is shown in Fig. 1 with isophotes. These structures are typical for the filamentary CNM described by Kalberla et al. (2016). 


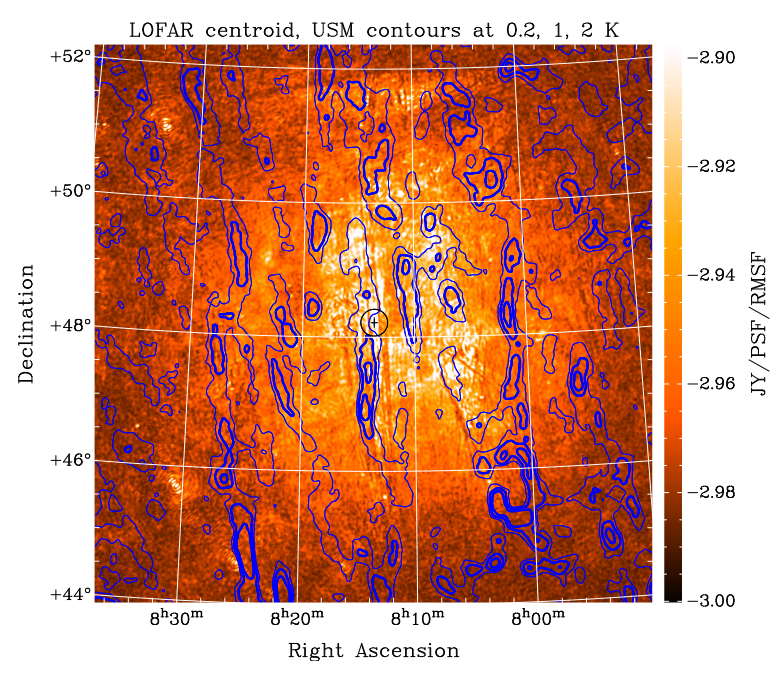

Fig. 2. Centroid map (Faraday depth in $\mathrm{rad} \mathrm{m}^{-2}$ ) in ecliptic coordinates derived from the LOFAR polarized emission in direction to 3C 196. The filamentary features from an HI USM map at $v_{\mathrm{LSR}}=-3.76 \mathrm{~km} \mathrm{~s}^{-1}$ are overlaid with contours of $0.2,1$, and $2 \mathrm{~K}$. The position of 3C 196 in the field center is indicated.

For a more detailed comparison between HI filaments and the polarized emission we calculate the centroid map from the LOFAR data cube of polarized emission at various Faraday depths. Such a centroid map should be sensitive to shifts in Faraday depth, caused by foreground filaments. In Fig. 2 we display this centroid map, overlaid with CNM filaments (red contours) from the EBHIS HI USM database at a velocity of $v_{\text {LSR }}=-3.76 \mathrm{~km} \mathrm{~s}^{-1}$. We find most of the HI filaments aligned with dips (dark blue) in the LOFAR centroid map but there is no unambiguous one-to-one correlation, even when checking the $\mathrm{CNM}$ at other velocities. However a comparison between HI gas and LOFAR data shows in general a good alignment between CNM filaments and polarized structures in Faraday depth. Moreover, Zaroubi et al. (2015) find a very clear correlation between the filamentary structures, detected with LOFAR, and the magnetic field orientation, probed by the Planck satellite. This is consistent with Kalberla et al. (2016) who report that there is in general a good agreement between CNM filaments and the magnetic field direction, probed by Planck.

After comparing LOFAR, EBHIS and Planck data at $353 \mathrm{GHz}$ we suspect that LOFAR filaments in polarized emission and HI are related to each other. Both probe the magnetic field as observed by Planck. We consider therefore the 3C 196 field as a prime target to study the relations between magnetic field orientation and induced anisotropic turbulence in the HI gas.

\section{Preparing the data for analysis}

\subsection{Database}

We use data from the first EBHIS data release (Winkel et al. 2016a). This survey covers all declination $\delta>-5^{\circ}$. The rms uncertainties of the brightness temperatures per channel are $90 \mathrm{mK}$ at a full width at half maximum (FWHM) width of $\delta v=1.44 \mathrm{~km} \mathrm{~s}^{-1}$. From the original EBHIS database, corrected for instrumental baselines, radio interference and stray radiation, we generated FITS data cubes, covering a region of $10^{\circ} \times 10^{\circ}$ in equatorial coordinates with a rotational symmetric Gaussian beam of 10'8 FWHM beam-size (Winkel et al. 2016b).

\subsection{Spectral analysis}

To quantify anisotropies in the spectral power distribution it is necessary to derive unbiased angular dependencies. Calculating a map of the HI distribution demands restricting the range of the source distribution on the sphere by applying a window function. After Fourier transform one obtains a convolution of the transformed true distribution with the transform of the window function (Bracewell 2000). For a rectangular window this causes a cross-like structure in the UV plane, with a particular nasty strong response at low spatial frequencies. To mitigate this effect the image data are usually apodized at the field boundaries with a $\sim 5$ pixel wide cosine function, afterwards the median is used to calculate the power spectrum, avoiding strong biases from the cross (e.g., Martin et al. 2015).

This recipe mitigates effects from the window response in case of average power spectra. When deriving an angular power distribution we found that the artificial cross structure is still dominant. To overcome this bias, a strictly spherical apodization with a strong taper is needed. It is further desirable to use a large untapered region but with low sidelobes of the window function in the Fourier domain. After some tests we have chosen a rotational symmetric $50 \%$ cosine taper (Tukey) window (Harris 1978), leaving the central $50 \%$ of the image untapered and applying then a cosine taper that reaches zero at the field boundary. In the UV plane this taper function leads to a slight smoothing, similar to the smoothing caused by the Fourier transform of the primary beam function in case of interferometer observations. The first sidelobe of the Fourier transformed Tukey taper is at a level of $-20 \mathrm{~dB}$ (see Fig. 31, Harris 1978).

In the following we demonstrate the individual steps is our data processing. In Fig. 1 we indicate with yellow circles the inner untapered $50 \%$ and the outer range $(100 \%)$ where the data are tapered to zero. In Fig. 3 we display on the left side the original image of the HI distribution at $v_{\mathrm{LSR}}=-3.76 \mathrm{~km} \mathrm{~s}^{-1}$. On the right the same after apodization with a 50\% Tukey taper. In analogy to a synthesis radio telescope, this image resembles a primary beam attenuated image. Figure 4 displays the power distribution, the squared moduli of the image after Fourier transform. Here we used for display a logarithmic representation, at the left without and at the right with apodization.

The EBHIS maps are gridded with a Gaussian kernel. Data processing as well as smoothing due to the telescope beam result in an effective Gaussian beam of 10'8. A deconvolution is thus necessary. After Fourier transform we divide the derived 2D power distribution by the square of the transformed beam response. The result is shown in Fig. 5. On the right is the 2D power spectrum that we use for further analysis. It is obvious from this figure. that high spatial frequencies are severely affected by instrumental noise that was amplified by the beam response function. This contribution can safely be considered as isotropic, without position angle dependent biases.

\section{Deriving the spectral index}

After generation of HI maps, subsequent apodization and Fourier transform with correction for beam convolution as explained in the previous section, we proceed with the data analysis.

\subsection{Average power spectrum and noise}

To determine the average (isotropic) power spectrum we integrate the $2 \mathrm{D}$ power distribution in annuli of constant spatial 

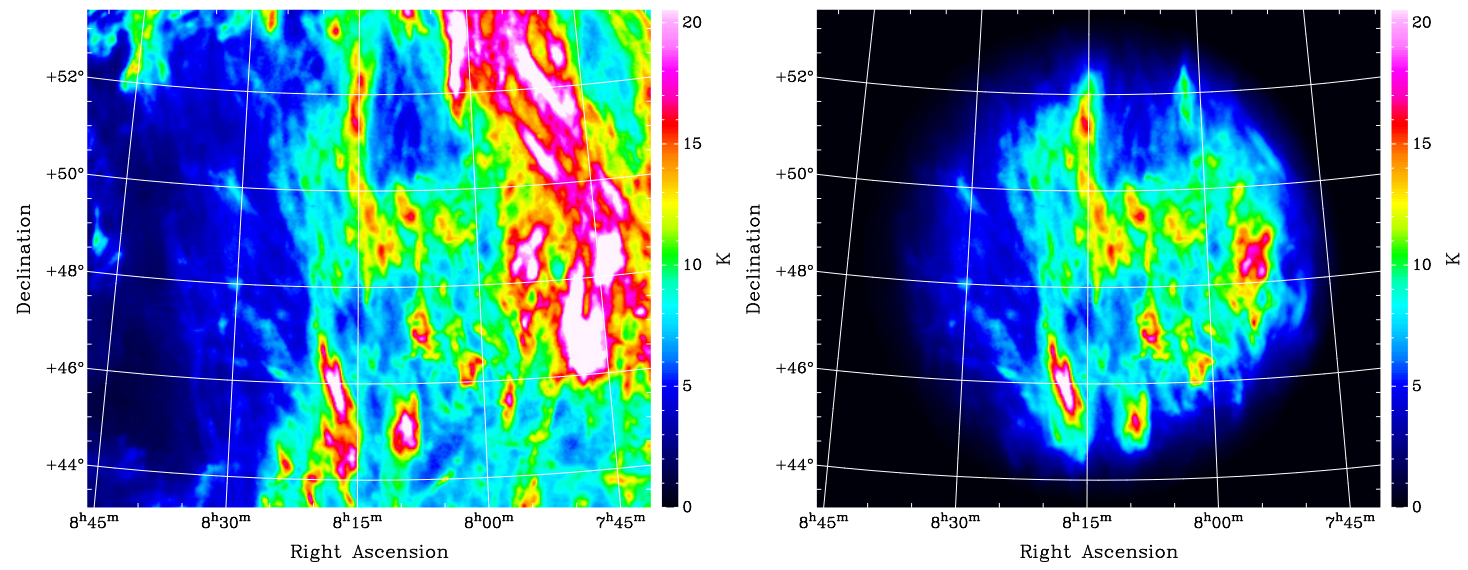

Fig. 3. Left: $\mathrm{HI} T_{\mathrm{B}}$ distribution at $v_{\mathrm{LSR}}=-3.76 \mathrm{~km} \mathrm{~s}^{-1}$ as observed; right: $\mathrm{HI} T_{\mathrm{B}}$ distribution after apodization with a $50 \%$ Tukey taper.
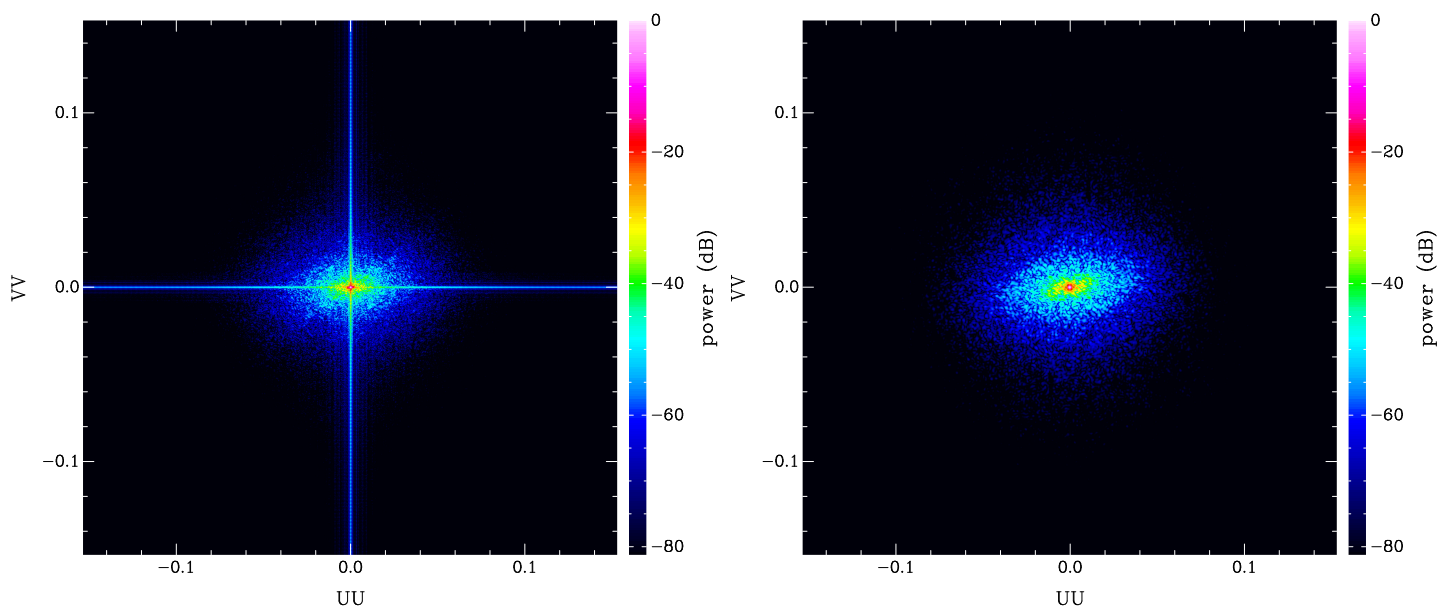

Fig. 4. Left: normalized power distribution at $v_{\text {LSR }}=-3.76 \mathrm{~km} \mathrm{~s}^{-1}$ as observed; right: HI power distribution of the apodized HI map. The scales are logarithmic in $\mathrm{dB}$.
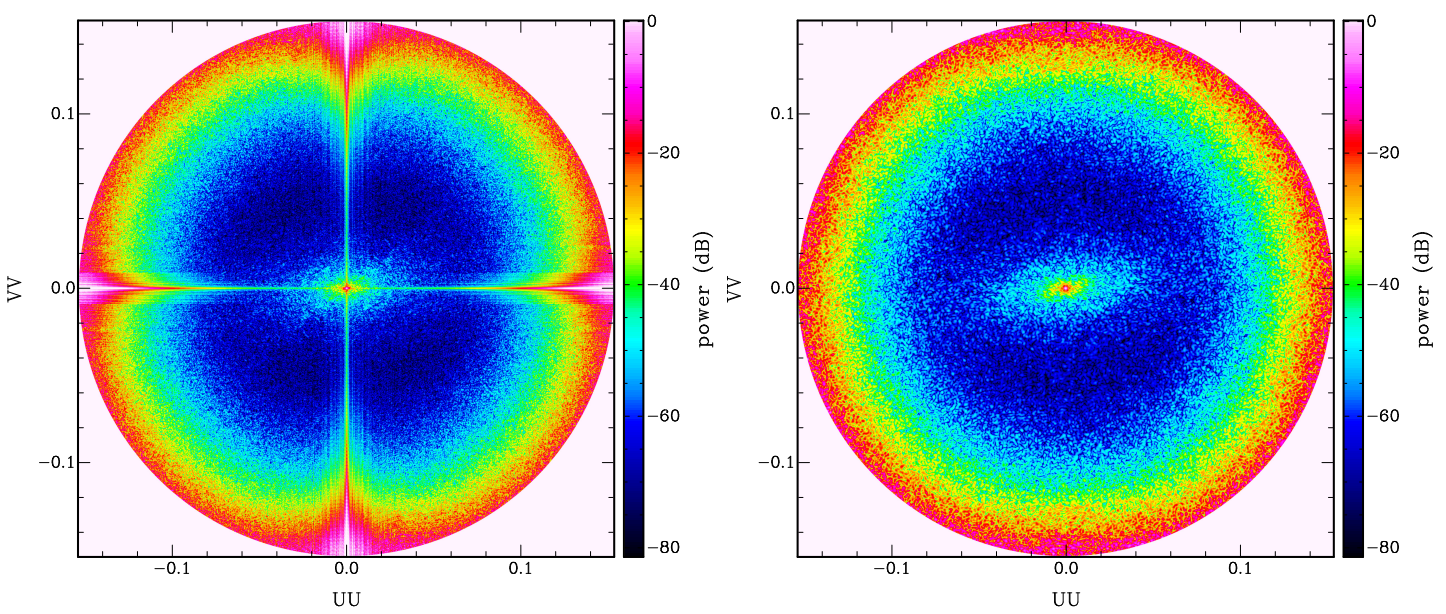

Fig. 5. Left: normalized power distribution at $v_{\mathrm{LSR}}=-3.76 \mathrm{~km} \mathrm{~s}^{-1}$, beam corrected; right: HI power distribution of the beam corrected apodized HI map. The scales are logarithmic in $\mathrm{dB}$.

frequencies $k=\left(k_{x}^{2}+k_{y}^{2}\right)^{1 / 2}$. As an example we use here the data from Fig. 5 (right panel) at $v_{\mathrm{LSR}}=-3.76 \mathrm{~km} \mathrm{~s}^{-1}$. The result is plotted in Fig. 6 marked by black dots. We obtain an average power distribution $P(k)$ that can be described by the relation

$P(k)=c \cdot k^{\gamma}+N(k)$.
Here $c$ is an arbitrary scale factor, resulting from the fact that we normalize in all cases the total power of the $2 \mathrm{D}$ power maps. $\gamma$ is the spectral index and $N(k)$ the contribution due to instrumental noise. It is obvious from Fig. 5 (right panel) that the noise dominates at high spatial frequencies. This is also clearly visible in Fig. 6. 


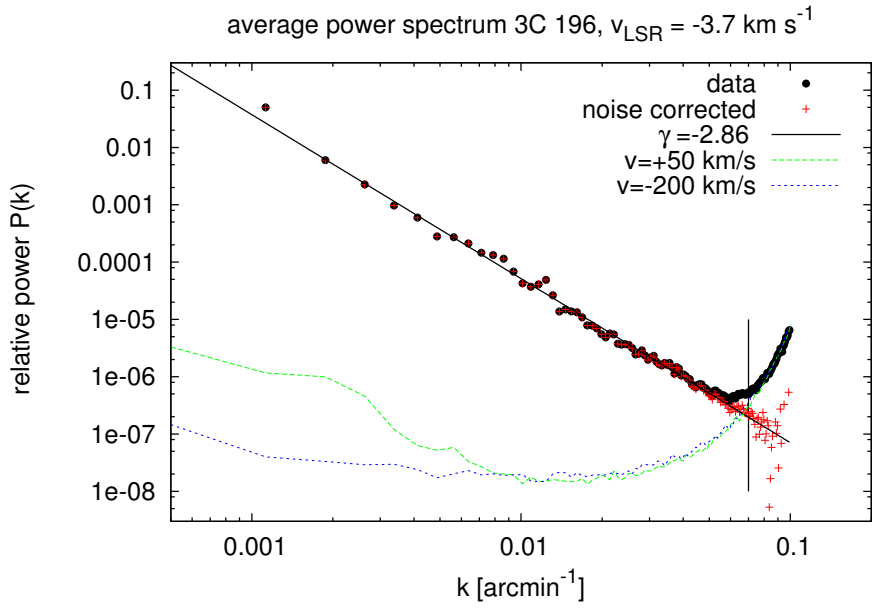

Fig. 6. EBHIS averaged angular power spectrum (black dots) at $v_{\mathrm{LSR}}=$ $-3.76 \mathrm{~km} \mathrm{~s}^{-1}$. Systematic and statistical uncertainties are estimated quantitatively by the power spectra calculated for emission free portion of the EBHIS data at $v_{\mathrm{LSR}}=+50 \mathrm{~km} \mathrm{~s}^{-1}$ (green) and $v_{\mathrm{LSR}}=-200 \mathrm{~km} \mathrm{~s}^{-1}$ (blue). Data after subtraction of a noise template $N(k)$ are plotted in red. The distribution for $k<0.07 \mathrm{arcmin}^{-1}$ (vertical line) is approximated by a power-law with $\gamma=-2.86 \pm 0.03$.

The noise contribution $N(k)$ to the power spectrum is in principle unknown, but can easily be estimated from channel maps without HI emission. In Fig. 6 we plotted as examples the noise power measured at $v_{\mathrm{LSR}}=+50 \mathrm{~km} \mathrm{~s}^{-1}$ and $v_{\mathrm{LSR}}=$ $-200 \mathrm{~km} \mathrm{~s}^{-1}$ after matching the power spectra at high spatial frequencies. A slight matching of the noise power is necessary because the system noise is not constant across the observed band, also the system noise increases with line temperature.

The observations suffer from uncertainties caused by radio frequency interference (RFI) and residual baseline uncertainties, including remaining errors in the correction for stray radiation, see Winkel et al. (2016a) for discussion. The noise spectra in Fig. 6 differ predominantly for $k<0.008 \operatorname{arcmin}^{-1}$ corresponding to scales larger than $2^{\circ}$ and are caused by such systematic uncertainties. The two examples in Fig. 6 represent approximately the range of expected uncertainties for EBHIS observations. Using 40 emission free channels we derive a noise template $N(k)$. Subtracting $N(k)$ we derive the unbiased normalized power spectrum $P_{\mathrm{av}}(k)=k^{\gamma}$, plotted in Fig. 6 in red, and fit the spectral index $\gamma=-2.86 \pm .03$ using all spatial frequencies $k<0.07 \operatorname{arcmin}^{-1}$.

It is obvious from Fig. 6 (red) that uncertainties in the derived power spectrum increase rapidly for $k \gtrsim 0.08 \mathrm{arcmin}^{-1}$. This limit depends on the signal-to-noise ratio of the observations but reflects also the limited spatial sensitivity of the $100-\mathrm{m}$ telescope. For our analysis in the 3C 196 field we strictly use in the following only data at spatial frequencies $k<0.07 \mathrm{arcmin}^{-1}$. This limit is indicated in all plots with a horizontal line.

\subsection{Angular power distribution}

To determine anisotropies, we first search for an average position angle. We average data within sectors $\Phi \pm \delta \Phi$ to obtain the power $P(\Phi, k)$ at position angles $\Phi$. Some care is necessary here, since we need enough samples per sector for statistical significant results. Position angles are ambiguous by $180^{\circ}$. Dividing this range into $n$ sectors implies that the noise increases by a factor of $\sqrt{n}$.

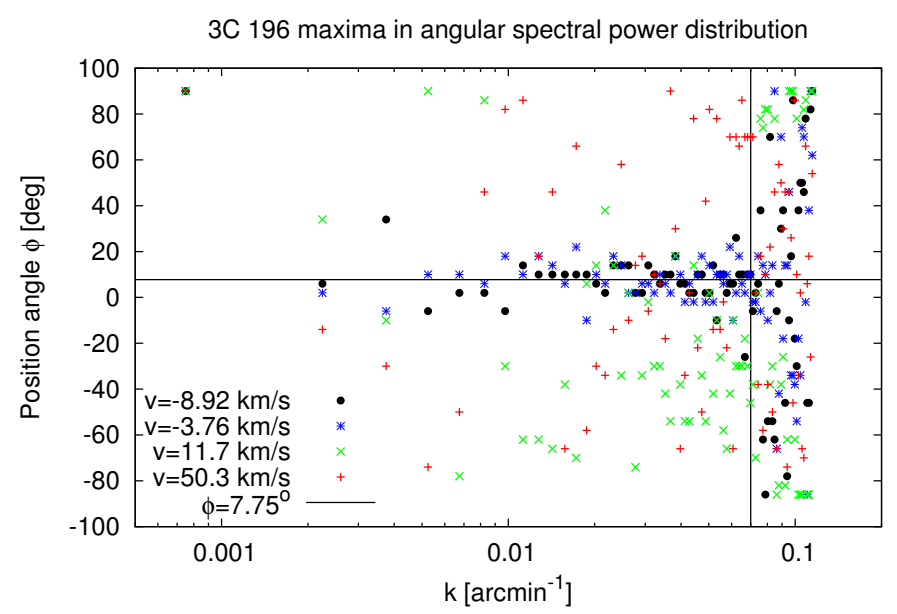

Fig. 7. Angular peak power distribution for single channels at velocities of $v_{\mathrm{LSR}}=-8.92,-3.76,11.69$, and $50.3 \mathrm{~km} \mathrm{~s}^{-1}$. Data for $k>$ $0.07 \mathrm{arcmin}^{-1}$ (vertical line) are affected by the instrumental noise. The average position angle for $v_{\mathrm{LSR}}=-8.92$ and $-3.76 \mathrm{~km} \mathrm{~s}^{-1}$ is $\phi=7.5$.

Searching for an average position angle over a range of spatial frequencies, this amplification is balanced by averaging over $m$ spatial frequencies, the formal increase of the uncertainties is then $\sqrt{n / m}$. After some tests we have chosen $n=45$, since the typical number of samples in spatial frequencies turned out to be $m \sim 23$, hence $\sqrt{n / m} \sim \sqrt{2}$ with our choice of $2 \delta \Phi=4^{\circ}$. Thus the selection of $\delta \Phi$ is not critical as long as position angles are correlated over a certain range in spatial frequencies.

Figure 7 displays some characteristic results of our search for anisotropies. This plot displays the position angle $\Phi$ for the peak power emission. For two channels at velocities $v_{\mathrm{LSR}}=-8.92$ and $-3.76 \mathrm{~km} \mathrm{~s}^{-1}$ we find a rather narrow and well defined distribution for the peaks around an average of $\Phi=7.5$ for spatial frequencies between $0.005<k<0.05 \operatorname{arcmin}^{-1}$ (black and blue points). The channel map at $v_{\mathrm{LSR}}=11.69 \mathrm{~km} \mathrm{~s}^{-1}$ (green) has quite a different angular power distribution with an average at $\Phi \sim-45^{\circ}$, but with a large scatter. We also display the distribution for a $v_{\mathrm{LSR}}=50 \mathrm{~km} \mathrm{~s}^{-1}$ (red), this case shows a random scatter. The distribution at high spatial frequencies, $k>0.07 \operatorname{arcmin}^{-1}$ is in general very noisy and the first data point at $k=0.00075 \mathrm{arcmin}^{-1}$ is affected by smoothing from the apodization function.

We analyse henceforth data in the range $0.005<k<$ $0.05 \mathrm{arcmin}^{-1}$ for an automated determination of anisotropies in the spectral power distribution and fit average position angles for all velocity channels. Figure 8 shows for velocities $-11.5<v_{\mathrm{LSR}}<-1.2 \mathrm{~km} \mathrm{~s}^{-1}$ a constant position angle with a typical dispersion of $8 \mathrm{~km} \mathrm{~s}^{-1}$ and a weighted mean of $\Phi=6.9$. This angle corresponds to the position angle $\Phi_{\text {mag }} \sim 97^{\circ}$ of the HI filaments, shown in Fig. 2 and is aligned with the magnetic field (Zaroubi et al. 2015). Thus we use in the Fourier plane $\Phi_{\perp}=7.5$.

We can draw here our first conclusion about the nature of the observed MHD turbulence. According to Kandel et al. (2016) Alfvén and slow modes cause the eddies to be elongated in direction parallel to the sky-projected mean magnetic field, while for fast modes the elongation is perpendicular. We find elongation parallel to the magnetic field, hence no evidence for fast modes. 


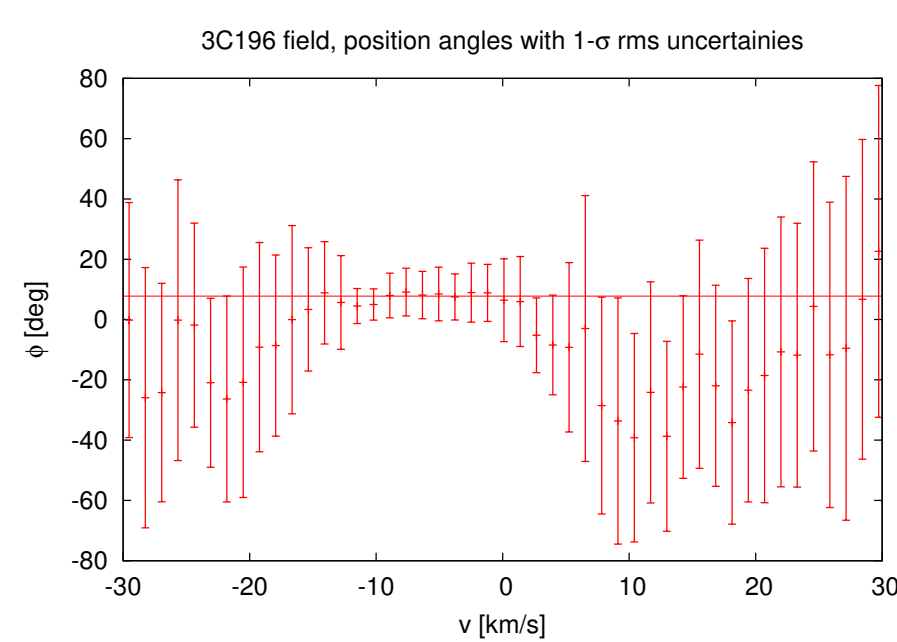

Fig. 8. Position angles fit for each channel at spatial frequencies $0.005<$ $k<0.05$. The average position angle $\phi=7.5$ from Fig. 7 is indicated with a horizontal line.

\subsection{Anisotropic power spectra}

We verified that the peaks in the angular power distribution are associated with minima in the power distribution at angles offset by typically $90^{\circ}$. We define for each velocity channel $v_{\mathrm{LSR}}$ the power anisotropy

$$
\begin{aligned}
Q\left(k, v_{\mathrm{LSR}}\right) & =P\left(\Phi_{\perp}, k, v_{\mathrm{LSR}}\right) / P\left(\Phi_{\|}, k, v_{\mathrm{LSR}}\right) \\
& =P_{\perp}\left(k, v_{\mathrm{LSR}}\right) / P_{\|}\left(k, v_{\mathrm{LSR}}\right),
\end{aligned}
$$

such that $Q=1$ denotes isotropy, while larger values of $Q$ mean larger anisotropy with eddies elongated parallel to the magnetic field. We calculate $Q$ by integrating in sectors $0^{\circ}<\Phi_{\perp}<15^{\circ}$ and $90^{\circ}<\Phi_{\|}<105^{\circ}$.

The anisotropies $Q\left(k, v_{\mathrm{LSR}}\right)$ show quite some scatter. We define therefore a geometric mean anisotropy

$Q_{\mathrm{aver}}\left(v_{\mathrm{LSR}}\right)=\exp \left[\frac{1}{n} \sum_{i=1}^{n} \ln Q\left(k_{i}, v_{\mathrm{LSR}}\right)\right]$,

for $n$ data-points in the range $0.005<k_{i}<0.05 \mathrm{arcmin}^{-1}$. $Q_{\text {aver }}\left(v_{\mathrm{LSR}}\right)$ is close to the median anisotropy $Q_{\text {median }}\left(v_{\mathrm{LSR}}\right)$ for this spatial frequency range.

Figures 9 and 10 show power spectra at $v_{\mathrm{LSR}}=$ $-3.76 \mathrm{~km} \mathrm{~s}^{-1}$ and $v_{\mathrm{LSR}}=-8.92 \mathrm{~km} \mathrm{~s}^{-1}$ with rather strong average anisotropies, $Q_{\text {aver }}=12.1$ and 18.8. Particular strong local anisotropies are visible in Fig. 9. For comparison we add in Fig. 11 a plot for $v_{\mathrm{LSR}}=11.69 \mathrm{~km} \mathrm{~s}^{-1}$, a case without significant anisotropy $\left(Q_{\mathrm{aver}}=1.8\right)$, see also Fig. 7 (green).

To summarize our finding from Figs. 9 to 11, we find significant anisotropies at channels with velocities around $v_{\mathrm{LSR}} \sim$ $-4 \mathrm{~km} \mathrm{~s}^{-1}$ in contrast to $v_{\mathrm{LSR}} \sim 11 \mathrm{~km} \mathrm{~s}^{-1}$, where no significant anisotropies are detected. Except for some deviations in the range $0.005 \lesssim k \lesssim 0.02 \mathrm{arcmin}^{-1}$, the red and green power spectra appear in logarithmic presentation just to be displaced from the isotropic distribution, sharing a similar slope but on average with slight inclinations in opposite directions.

\subsection{HI parameter dependencies on $v_{\mathrm{LSR}}$}

Figure 12 summarizes global properties of the HI gas distribution in the 3C 196 field. To characterize the HI emission we calculate the average brightness temperature as the weighted mean

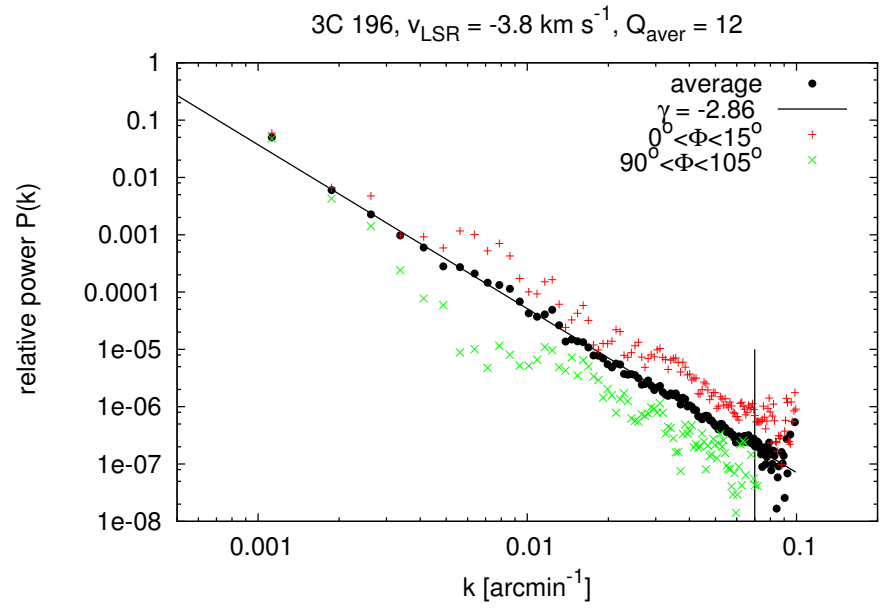

Fig. 9. Average power spectrum observed for $v_{\mathrm{LSR}}=-3.76 \mathrm{~km} \mathrm{~s}^{-1}$ and fit power law (black) with $\gamma=-2.86 \pm 0.03$ for $k<0.07 \mathrm{arcmin}^{-1}$ (vertical line). In addition the power spectrum for $0^{\circ}<\Phi<15^{\circ}$ (red) and $90^{\circ}<\Phi<105^{\circ}$ (green) is given. The average anisotropy factor is $Q_{\text {aver }}=12.1$.

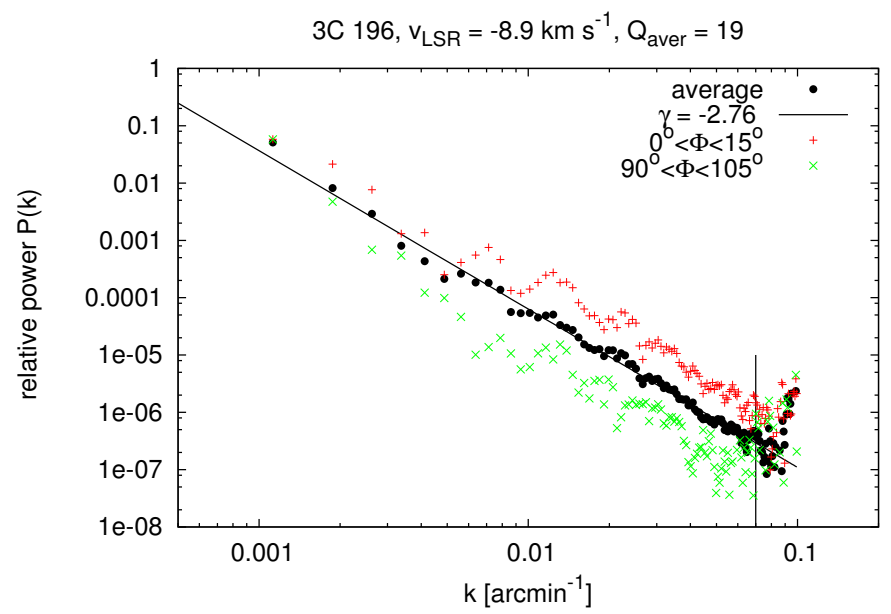

Fig. 10. Average power spectrum observed for $v_{\mathrm{LSR}}=$ $-8.92 \mathrm{~km} \mathrm{~s}^{-1}$ (black dots) and fit power law with $\gamma=-2.76 \pm 0.04$ for $k<0.07 \mathrm{arcmin}^{-1}$ (vertical line). In addition the power spectrum for $0^{\circ}<\Phi<15^{\circ}$ (red) and $90^{\circ}<\Phi<105^{\circ}$ (green) is given. The average anisotropy factor is $Q_{\text {aver }}=18.8$

over the apodized brightness temperature,

$T_{\mathrm{B} \text { aver }}=\frac{\sum T_{\mathrm{B}} \cdot W}{\sum W}$

Here $W$ is the apodization weight due to the $50 \%$ Tukey taper function. The HI distribution (plotted in red) shows two major components in emission, peaking at $v_{\mathrm{LSR}}=-1.2 \mathrm{~km} \mathrm{~s}^{-1}$ and at $v_{\mathrm{LSR}}=13 \mathrm{~km} \mathrm{~s}^{-1}$. For comparison we plot also the derived anisotropy $Q_{\text {aver }}\left(v_{\text {LSR }}\right)$ (blue) and the average spectral in$\operatorname{dex} \gamma\left(v_{\mathrm{LSR}}\right)$ (green). Anisotropies are strongest for channels with best defined position angles (compare Fig. 8 with Fig. 12). The major local minima in the spectral index distribution $\gamma$ coincide with the peaks of the HI components. A similar trend was found by Stanimirovic et al. (1999) in the SMC.

The velocities of CNM components tend to be closely related to the center velocities of the warm neutral medium (WNM; Kalberla et al. 2016). It is also well established that the velocity dispersion of the WNM is significantly larger than the dispersion of the CNM components (e.g., Heiles \& Troland 2003). The 


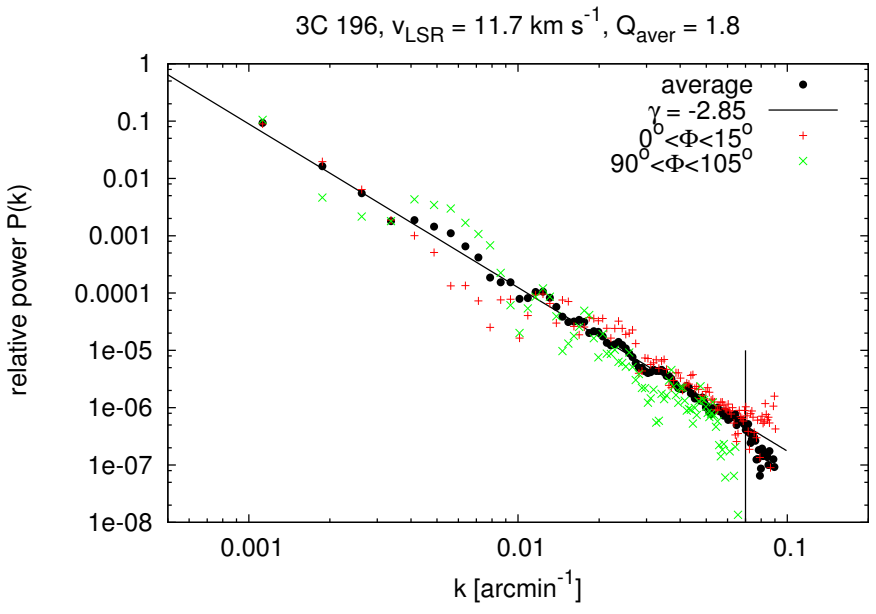

Fig. 11. Average power spectrum observed for $v_{\mathrm{LSR}}=$ $11.69 \mathrm{~km} \mathrm{~s}^{-1}$ (black dots) and fit power law with $\gamma=-2.85 \pm 0.03$ for $k<0.07 \operatorname{arcmin}^{-1}$ (vertical line). In addition the power spectrum for $0^{\circ}<\Phi<15^{\circ}$ (red) and $90^{\circ}<\Phi<105^{\circ}$ (green) is given. The average anisotropy factor is $Q_{\text {aver }}=1.8$.

CNM is clumpy while the WNM has a diffuse structure. Thus the CNM adds extra power at high spatial frequencies and for a mix of CNM and WNM gas one expects that the spectral index should be flattest close to the center velocity of the WNM component where the CNM is most pronounced. Correspondingly Martin et al. (2015), after decomposing WNM and CNM components, find for the column density distribution that the spectral index of the CNM is shallower than that of the WNM.

For the observed mix of WNM and CNM we find the opposite. Consistent with Stanimirovic et al. (1999), the spectral index for thin slices in velocity is steepest at the center velocity of the WNM component. Figure 13 shows that the spectral index steepens as the CNM Doppler temperature (hence the velocity dispersion) decreases. We find no indications for a break in the spectral power distribution that could be attributed to either the CNM or the WNM. This suggests that turbulence in the WNM and CNM cannot be considered independently. Turbulence may induce the formation of CNM when the WNM is pressurized and put in a thermally unstable state (Saury et al. 2014). Since turbulence is probably playing a major rôle in regulating the ratio between WNM and CNM, phase transitions must be the key in understanding the steepening of the spectral index. The usual assumption is that steep spectra correspond to random fields dominated by large-scale fluctuations (Lazarian \& Pogosyan 2000). In our case the HI filaments, visible over many degrees, may be indicative for an event that could have driven phase transitions.

The anisotropy measure $Q_{\mathrm{aver}}$, does not correlate with $T_{\mathrm{B} \text { aver }}$, nor with $\gamma$. Kalberla et al. (2016) found that the CNM is mostly organized in cold HI filaments with Doppler temperatures $T_{\mathrm{D}} \sim$ $223 \mathrm{~K}$. To check, whether $T_{\mathrm{D}}$ might be correlated with $\gamma$ or $Q_{\mathrm{aver}}$, we determined the geometric mean $T_{\mathrm{D}}$ for the CNM filaments toward the 3C 196 field. Again we find no correlation with $Q_{\text {aver }}$, but Fig. 13 shows that for the coldest parts of the filaments also the spectral index $\gamma$ (green) is steep.

HI absorption line data against the continuum source 3C 196 show two components, at a velocity of $v_{\mathrm{LSR}}=-2.2 \mathrm{~km} \mathrm{~s}^{-1}$ with an optical depth of $\tau=0.086$ and at $v_{\mathrm{LSR}}=15.3 \mathrm{~km} \mathrm{~s}^{-1}$ with $\tau=0.130$. The component at $v_{\mathrm{LSR}}=15.3 \mathrm{~km} \mathrm{~s}^{-1}$ has a spin temperature of $80.9 \mathrm{~K}$ (Mebold et al. 1982). This temperature compares well with the low geometric mean $T_{\mathrm{D}}$ determined by us for the whole field, but we note that we find the lowest

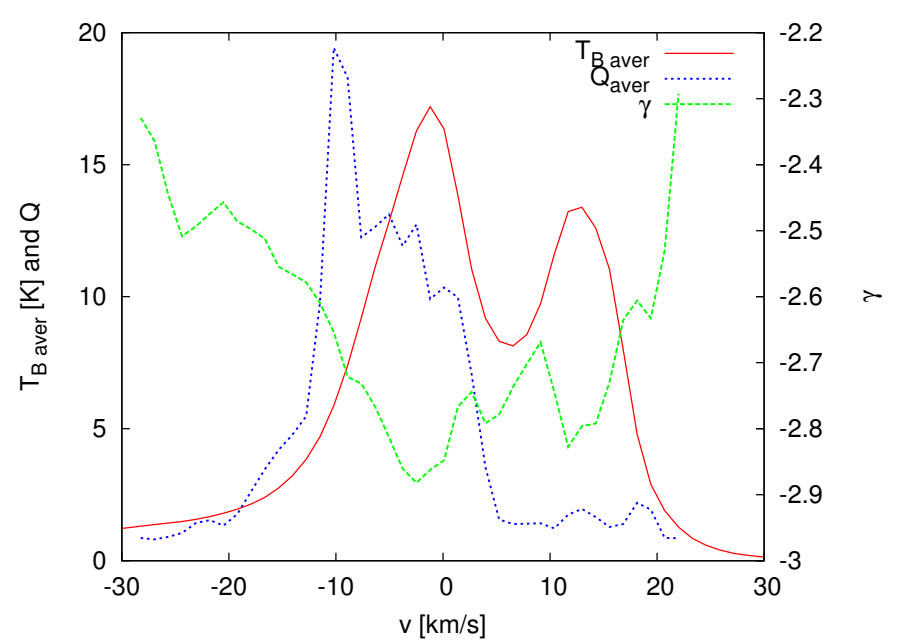

Fig. 12. Comparison between average brightness temperature $T_{\mathrm{B} \text { aver }}$ (red), anisotropy factor $Q_{\text {aver }}$ (blue), and spectral index $\gamma$ (green) for the 3C 196 field.

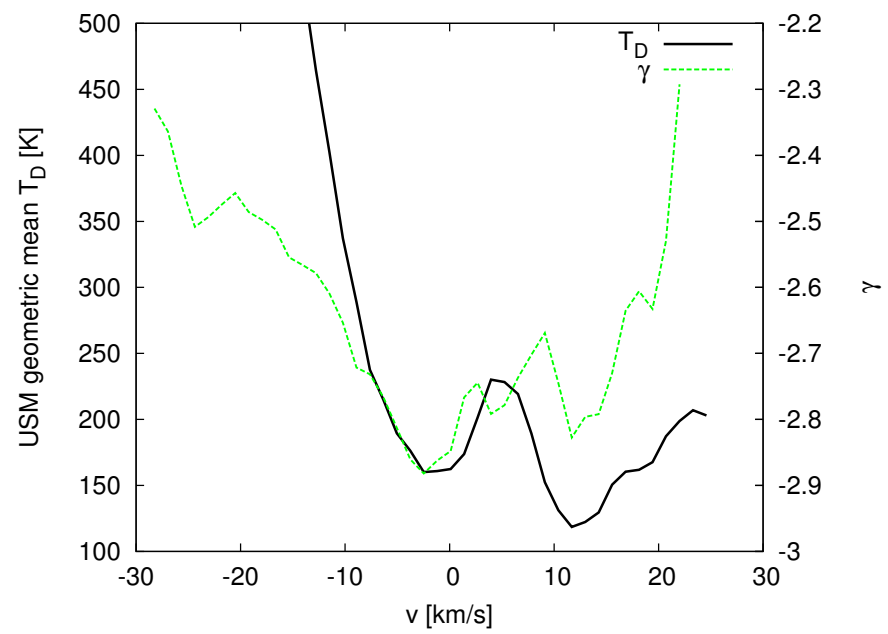

Fig. 13. Comparison between the geometric mean Doppler temperature $T_{\mathrm{D}}$ (black) and the spectral index $\gamma$ (green dashed) for the 3C 196 field.

geometric mean $T_{\mathrm{D}}$ at $v_{\mathrm{LSR}}=11.69 \mathrm{~km} \mathrm{~s}^{-1}$. Crovisier et al. (1978), using the Nançay telescope, find evidence for absorption at $v_{\mathrm{LSR}}=-7.0,-2.2$, and $15.7 \mathrm{~km} \mathrm{~s}^{-1}$. The additional component at $v_{\mathrm{LSR}}=-7.0 \mathrm{~km} \mathrm{~s}^{-1}$ most probably is due the fan beam of the Nançay telescope, extending in north/south direction and thus being more sensitive to filaments oriented in the same direction.

\subsection{Thick slices, velocity centroid}

So far we analyzed only individual channel maps, in each case covering a range of $\delta v_{\mathrm{LSR}}=1.44 \mathrm{~km} \mathrm{~s}^{-1}$ in velocity, the limiting resolution of the EBHIS (Winkel et al. 2016a). This channel width formally corresponds to a Doppler temperature of $T_{\mathrm{D}} \sim 50 \mathrm{~K}$. The median Doppler temperature of the CNM is $T_{\mathrm{D}} \sim 223 \mathrm{~K}$ (Kalberla et al. 2016). Figure 13 discloses that some of the filaments in the 3C 196 field are colder $\left(T_{\mathrm{D}} \gtrsim 160\right)$ than the median.

A velocity channel $\delta v_{\mathrm{LSR}}=1.44 \mathrm{~km} \mathrm{~s}^{-1}$ is, according to the definition of (Lazarian \& Pogosyan 2000), thin if the line-of-sight FWHM velocity width of the gas is larger than the channel width. Otherwise the observed slice is thick. Our observational setup implies that an individual velocity channel is thin 


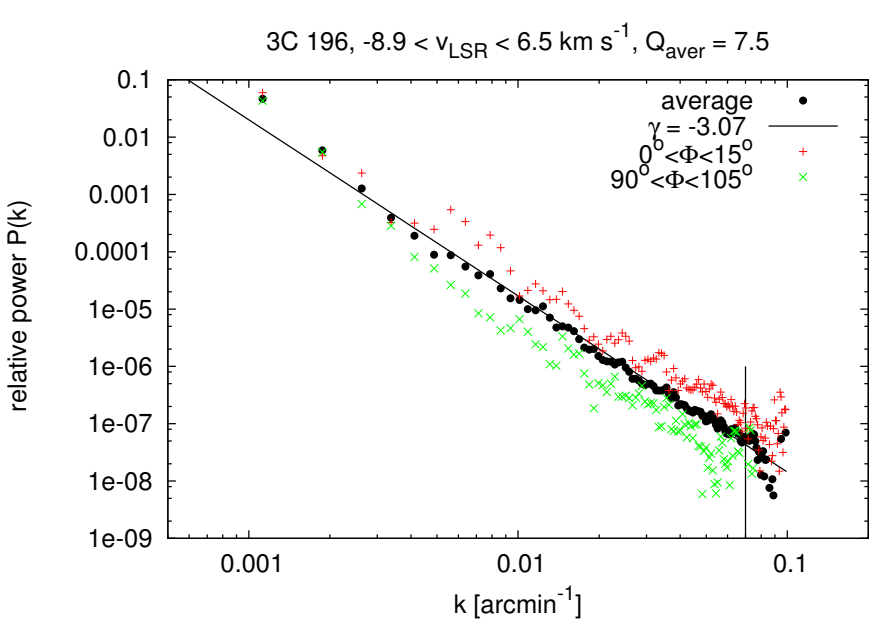

Fig. 14. Average power spectrum for a thick slice, $-8.9<v_{\mathrm{LSR}}<$ $6.5 \mathrm{~km} \mathrm{~s}^{-1}$ (black dots) and fit power law with $\gamma=-3.07 \pm 0.04$ for $k<0.07 \operatorname{arcmin}^{-1}$ (vertical line). In addition the power spectrum for $0^{\circ}<\Phi<15^{\circ}$ (red) and $90^{\circ}<\Phi<105^{\circ}$ (green) is given. The average anisotropy factor is $Q_{\text {aver }}=7.5$.

3C 196, velocity centroid for $-8.9<\mathrm{V}_{\mathrm{LSR}}<6.5 \mathrm{~km} \mathrm{~s}^{-1}, \mathrm{Q}_{\mathrm{aver}}=8.2$

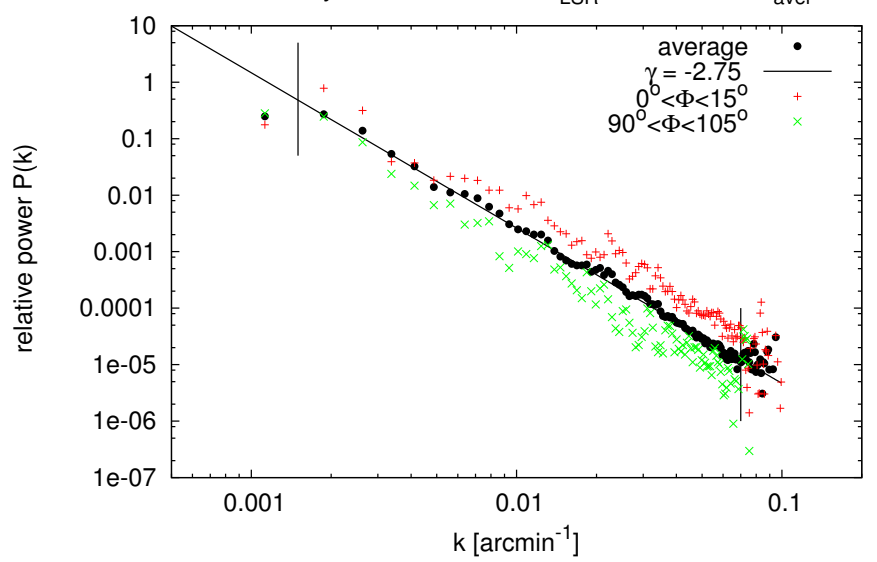

Fig. 15. Average power spectrum for the velocity centroid in the range $-8.9<v_{\text {LSR }}<6.5 \mathrm{~km} \mathrm{~s}^{-1}$ (black dots) and fit power law with $\gamma=$ $-2.75 \pm 0.04$ for $0.0015<k<0.07 \mathrm{arcmin}^{-1}$ (vertical lines). In addition the power spectrum for $0^{\circ}<\Phi<15^{\circ}$ (red) and $90^{\circ}<\Phi<105^{\circ}$ (green) is given. The average anisotropy factor is $Q_{\mathrm{aver}}=8.2$.

for most of the gas but in the limit it may be thick for the coldest CNM components.

Filamentary features are mostly sheets, seen tangentially, with a median thickness between 0.1 and $0.3 \mathrm{pc}$ (Heiles \& Troland 2005; Kalberla et al. 2016). Because the sheets are cold, we observe along the line of sight different radial velocities which causes in many cases an apparent positional shift perpendicular to the filament (see Figs. 10, 25, and 26 of Kalberla et al. 2016). The 3C 196 field under investigation contains several filaments (or sheets), approximately oriented parallel to each other.

The average HI emission contains two components. To cover the major part of the anisotropic filamentary range, we integrate over the channels for $-8.9<v_{\mathrm{LSR}}<6.5 \mathrm{~km} \mathrm{~s}^{-1}$ (see Fig. 12). This slice is thick (Lazarian \& Pogosyan 2000) with respect to the CNM but, because of blending with the isotropic component at $v_{\mathrm{LSR}} \sim 11 \mathrm{~km} \mathrm{~s}^{-1}$, does not cover the full width of the WNM as requested by Lazarian \& Pogosyan (2000) for a representative thick slice. A general problem with the HI distribution is that several HI components may be superposed along the line

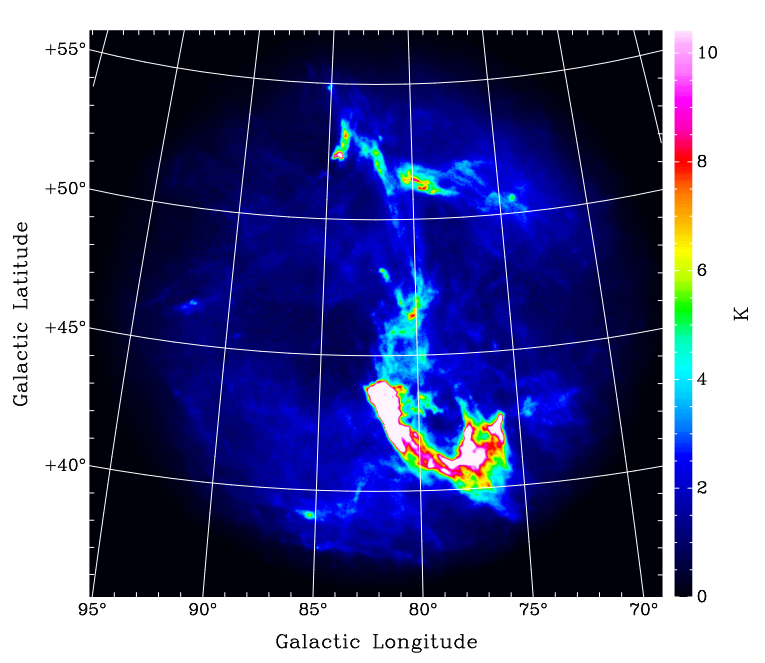

Fig. 16. Apodized HI distribution in direction to the FN1 field at a velocity of $v_{\text {LSR }}=3.97 \mathrm{~km} \mathrm{~s}^{-1}$. This map is used to test the methodology of our approach in case of diffuse HI structures.

of sight. An overlap in velocity may then, as in our case, smear out or merge individual $\mathrm{HI}$ features. In this case it is difficult to derive a representative thick slice. Figure 14 shows a power law $\gamma=-3.07 \pm 0.04$ with $Q_{\text {aver }}=7.5$. For the velocity centroid over the same velocity range we determine $\gamma=-2.75 \pm 0.04$, see Fig. 15 . The anisotropy is this case slightly larger, $Q_{\text {aver }}=8.2$.

According to Lazarian \& Pogosyan (2000, Eq. (28)), thin and thick sheets should have distinct different power indices. We find only a moderate steepening with slice thickness by $\delta \gamma \sim 0.2$, comparable to Stanimirović \& Lazarian (2001). Anisotropies are noticeable but weaker than for most of the prominent thin slices. Velocity centroids, proposed by Esquivel \& Lazarian (2005), are apparently not always the best choice when studying anisotropies.

\section{Diffuse $\mathrm{HI}$ structures at high Galactic latitudes}

The 3C 196 spectral component at $v_{\mathrm{LSR}} \sim 11 \mathrm{~km} \mathrm{~s}^{-1}$ shows in Fig. 11 only weak anisotropies with $Q_{\text {aver }}=1.8$. To get an estimate, how far power spectra and anisotropies in direction to the 3C 196 field deviate from typical diffuse HI structures, we decided to analyze for comparison a field without obvious filaments or anisotropies.

Miville-Deschênes \& Martin (2007) studied such a case, a very diffuse HI filament located in the extra-galactic window known as Firback North 1 (FN1, Dole et al. 2001) at high Galactic latitudes $\left(l=84^{\circ}, b=45^{\circ} .1\right)$. They used data from the Leiden/Dwingeloo Survey (LDS, Hartmann \& Burton 1997) and from the Green Bank Telescope (GBT) and calculated the integrated emission and the velocity centroid for $-86.2<v_{\mathrm{LSR}}<$ $47.8 \mathrm{~km} \mathrm{~s}^{-1}$. We are going to use this velocity range, representing a thick slice, for reference.

For the FN1 field we applied the same data-processing pipeline as described in Sect. 3. We used the EBHIS to generate a field with $38^{\circ}$ in diameter and apodized this with an inner diameter of $19^{\circ}$, see Fig. 16. We derived the angular power distribution and determined position angles for all emission channels. Figure 17 shows angles $45^{\circ} \gtrsim \phi \gtrsim 0^{\circ}$, but typically with a huge dispersion. The velocity range $-5 \lesssim v_{\mathrm{LSR}} \lesssim 10 \mathrm{~km} \mathrm{~s}^{-1}$ is outstanding, here we find better defined position angles, but with a systematical rotation by almost $40^{\circ}$. The position angles for 


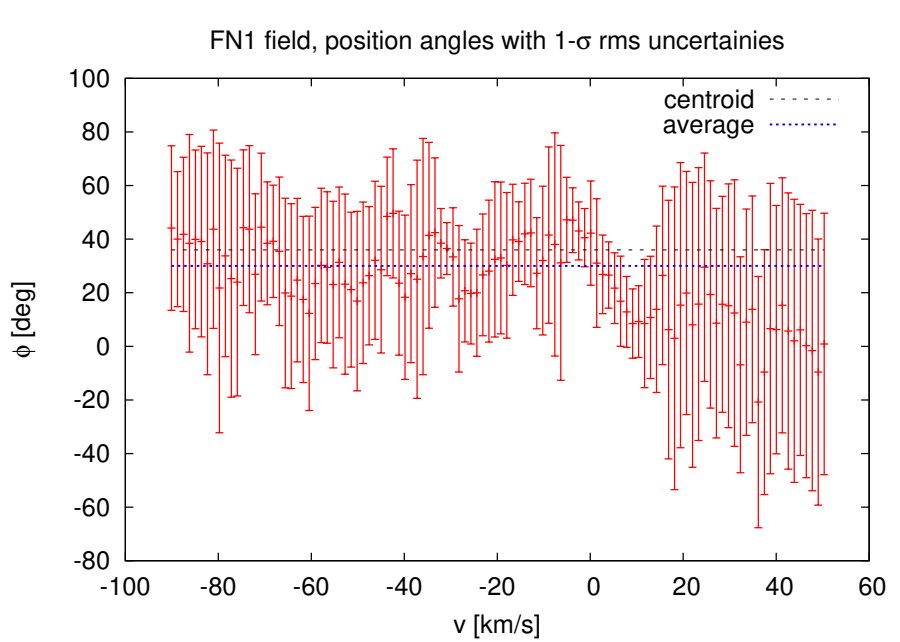

Fig. 17. Position angles fit for each channel at spatial frequencies $0.005<k<0.05$. The position angles for the centroid and the average emission, $\phi=36^{\circ} \pm 19^{\circ}$ and $\phi=30^{\circ} \pm 15^{\circ}$, respectively, are also indicated.

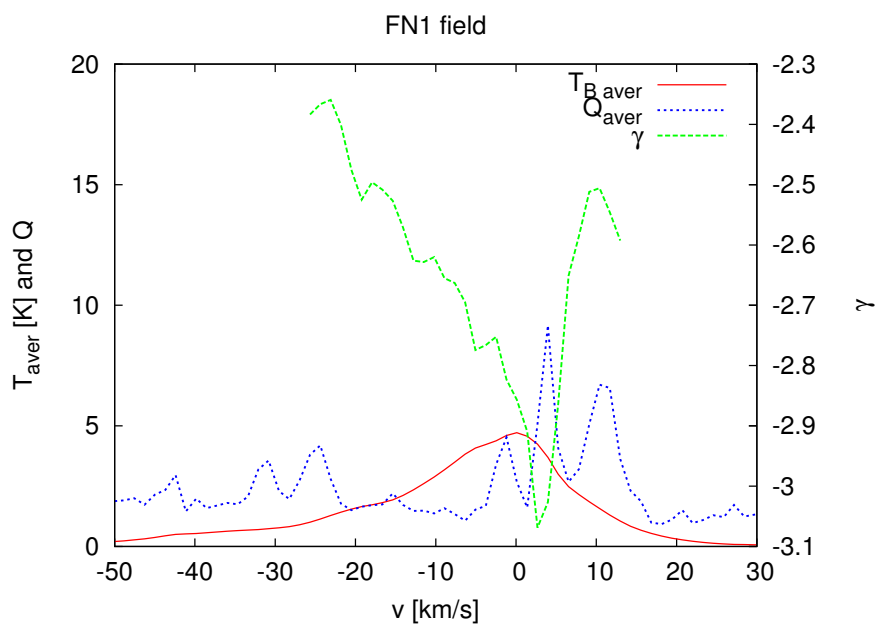

Fig. 18. Comparison between average brightness temperature $T_{\mathrm{B} \text { aver }}$ (red), anisotropy factor $Q_{\text {aver }}$ (blue), and spectral index $\gamma$ (green) for the FN1 field.

the centroid and the average emission are $\phi=36^{\circ} \pm 19^{\circ}$ and $\phi=30^{\circ} \pm 15^{\circ}$, respectively, also indicated in Fig. 17 .

Figure 18 displays the average emission (red) according to Eq. (4), average anisotropies (blue) and the fit average spectral index $\gamma$ (green). The emission is weak with a long extended tail to negative velocities; we plot only the most significant range $-50 \lesssim v_{\text {LSR }} \lesssim 30 \mathrm{~km} \mathrm{~s}^{-1}$. To avoid spurious results, we determined spectral indices $\gamma$ only for $T_{\mathrm{B} \text { aver }}>1 \mathrm{~K}$.

Close to the peak of the average emission $T_{\mathrm{B} \text { aver }}$, but shifted by two channels to positive velocities we find a remakable narrow minimum in the spectral index distribution. One more channel shifted to positive velocities, at $v_{\mathrm{LSR}}=3.97 \mathrm{~km} \mathrm{~s}^{-1}$, there is a narrow peak for $Q_{\text {aver }}$. We checked this channel in more detail and found anisotropies peaking at an angle of $\phi \sim 30^{\circ}$. The average power spectrum at this velocity is shown in Fig. 19, together with power spectra in direction of the derived position angle and perpendicular. There are marked anisotropies. In comparison to this single channel, representing a thin velocity slice, we find for the velocity centroid and the average emission, both determined

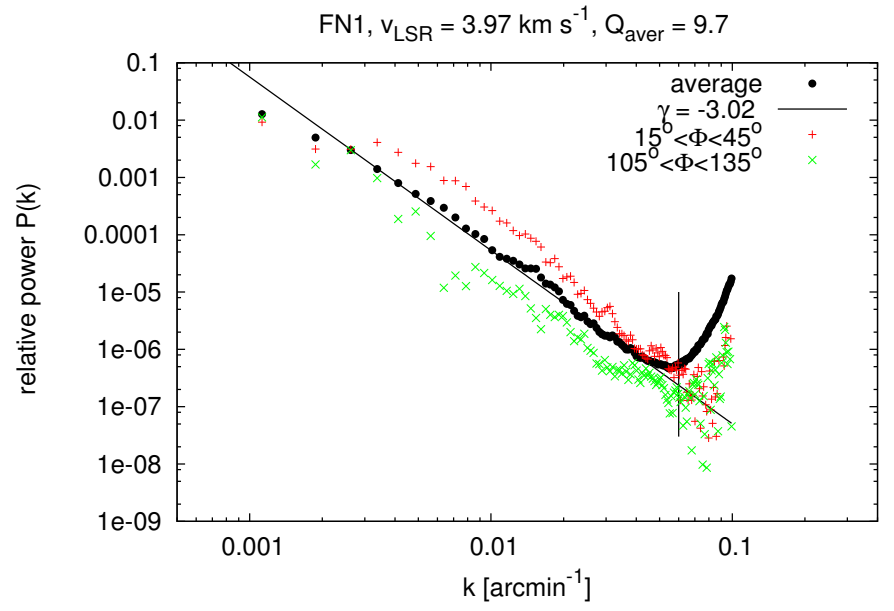

Fig. 19. Average power spectrum at $v_{\mathrm{LSR}}=3.97 \mathrm{~km} \mathrm{~s}^{-1}$ and fit power law (black) with $\gamma=-3.02 \pm 0.03$ for $k<0.06 \mathrm{arcmin}^{-1}$ (vertical line). In addition the power spectrum for $15^{\circ}<\Phi<45^{\circ}$ (red) and $115^{\circ}<\Phi<135^{\circ}$ (green) is given. The average anisotropy factor is $Q_{\text {aver }}=9.7$.

FN1 velocity centroid for $-86.2<\mathrm{v}_{\mathrm{LSR}}<47.8 \mathrm{~km} \mathrm{~s}^{-1}, \mathrm{Q}_{\mathrm{aver}}=2.2$

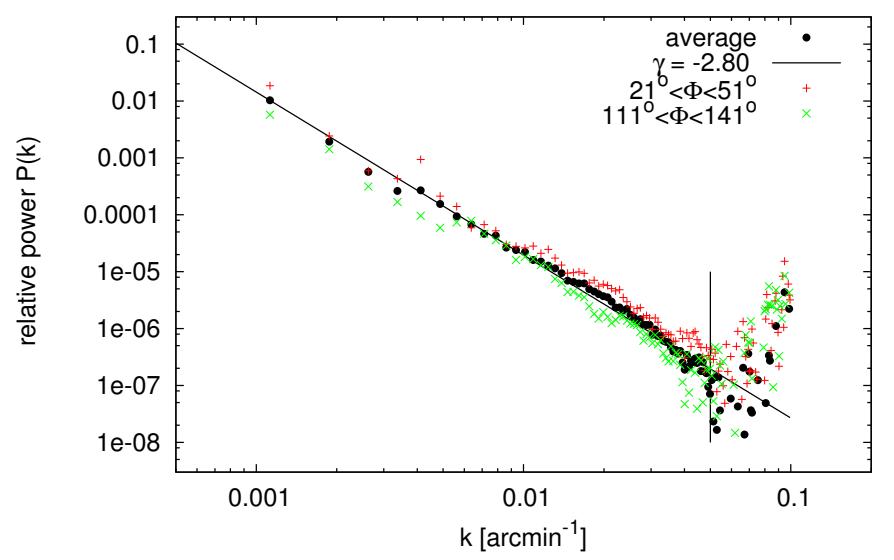

Fig. 20. Average power spectrum for the velocity centroid and fit power law (black) with $\gamma=-2.86 \pm 0.06$ for $k<0.05 \mathrm{arcmin}^{-1}$ (vertical line). In addition the power spectrum for $30^{\circ}<\Phi<60^{\circ}$ (red) and $120^{\circ}<\Phi<150^{\circ}$ (green) is given. The average anisotropy factor is $Q_{\text {aver }}=2.0$.

for $-86.2<v_{\mathrm{LSR}}<47.8 \mathrm{~km} \mathrm{~s}^{-1}$, only insignificant anisotropies $Q_{\text {aver }}=2.2$ (Figs. 20 and 21).

The spectral index for the velocity centroid is $\gamma=-2.80 \pm$ 0.03 and $\gamma=-2.97 \pm 0.02$ for the average emission profile. Miville-Deschênes \& Martin (2007) find for centroid and integrated emission $\gamma=-3.4$, inconsistent with our result. Their analysis might suffer from systematic biases, causing a steep spectral index. They deconvolved the LDS power distribution assuming a FWHM beam width of $30^{\prime}$ while the telescope beam is $36^{\prime}$. When generating maps there is some additional smoothing, depending on the methods used. Last not least, the LDS is not Nyquist sampled; this introduces some additional biases that cannot easily be specified.

We repeated our spectral analysis for the integrated emission omitting the beam correction. The result is plotted in Fig. 22 and should be compared with Fig. 21. The distribution at high spatial frequencies with a spectral steepening is a fraud, though it appears to be less noisy. When omitting a beam correction, the slope steepens from $\gamma=-2.97$ to $\gamma=-3.45$. We see that a subtle lapse in data processing may lead to significant biases. 


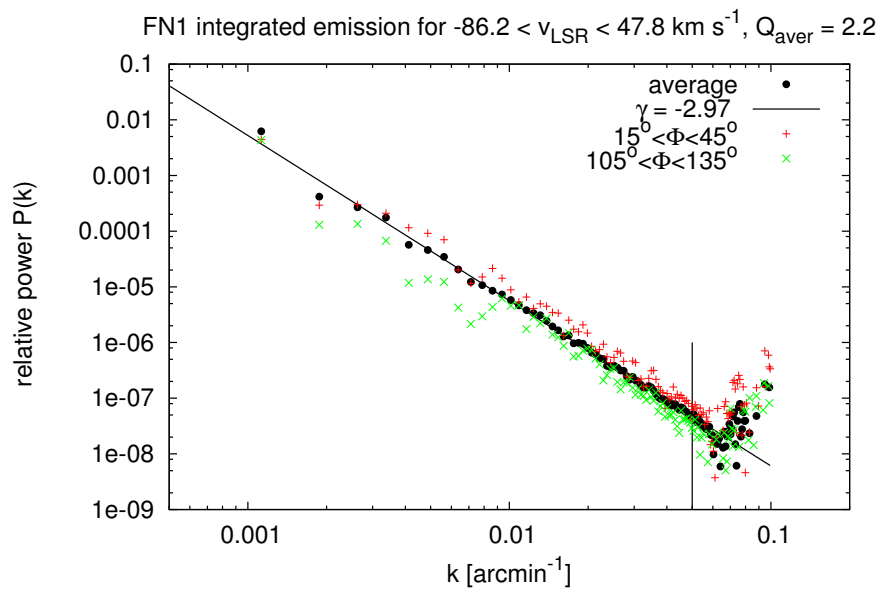

Fig. 21. Average power spectrum for the integrated emission and fit power law (black) with $\gamma=-3.00 \pm 0.03$ for $k<0.05 \mathrm{arcmin}^{-1}$ (vertical line). In addition the power spectrum for $30^{\circ}<\Phi<60^{\circ}$ (red) and $120^{\circ}<\Phi<150^{\circ}$ (green) is given. The average anisotropy factor is $Q_{\text {aver }}=2.0$.

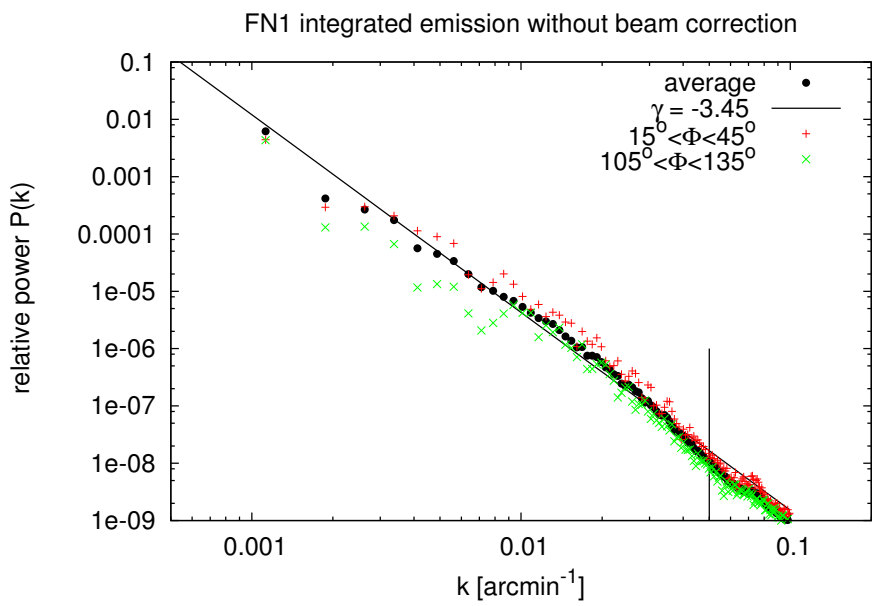

Fig. 22. To demonstrate biases in power distribution and derived spectral indices we repeat the analysis from Fig. 21 without a beam correction. The power spectrum at high spatial frequencies is biased.

Anisotropies at high spatial frequencies are suppressed. After reviewing the literature cited in Sect. 1 we note that it is not obvious whether in all cases a proper beam correction has been applied to the data. Some of the steep spectral indices might be due to this systematic bias, also a steepening of the power spectrum should be carefully investigated.

Recently Blagrave et al. (2016) reported a similar problem in the Ursa Major region. Miville-Deschênes et al. (2003) derive there a power law index $\gamma=-3.6 \pm 0.2$ for both, column density map as well as velocity centroid, while Blagrave et al. (2016) measured $\gamma=-2.68 \pm 0.14$ for column densities and $-2.54<\gamma<-2.42$ for the velocity centroid. We tested this case with EBHIS data and obtained for column densities and velocity centroid $\gamma=-2.68 \pm 0.07$, consistent with Blagrave et al. (2016).

Our conclusion after calculating power spectra and anisotropies for the FN1 field is that the 3C 196 anisotropies at $v_{\mathrm{LSR}} \sim-3.76 \mathrm{~km} \mathrm{~s}^{-1}$ are outstanding. For $-11.5<v_{\mathrm{LSR}}<$ $-1.2 \mathrm{~km} \mathrm{~s}^{-1}$ all filaments are aligned with the magnetic field. Anisotropies for the 3C 196 field at $v_{\mathrm{LSR}} \sim 11 \mathrm{~km} \mathrm{~s}^{-1}$ as well as for the two comparison fields are mostly around $Q_{\text {aver }} \sim 2$. However even in the FN1 field we find channels with strong anisotropies. The major difference is that in this case the anisotropies are restricted to narrow velocity intervals and the position angles change systematically. Checking the dust emission observed by Planck at $353 \mathrm{GHz}$ we find a pretty weak and noise limited signal without any indications for filamentary structures. Thus there is no clear evidence that one of the velocity channels is associated with a dust filament in this region. The power distribution at $v_{\mathrm{LSR}}=3.97 \mathrm{~km} \mathrm{~s}^{-1}$ will be discussed later in more detail. As long as further studies about anisotropies in the HI are missing, we consider $Q_{\text {aver }} \sim 2$ as typical for the unperturbed HI distribution. As "unperturbed" we understand regions that are not affected by obvious CNM filaments that can be seen in USM maps.

\section{Discussion}

After characterizing anisotropies for an unperturbed HI distribution, we return to a discussion of the 3C 196 field. We also compare observations with predictions from theoretical models.

To compare the derived power law index and the anisotropy measure $Q$ with anisotropies as expected for MHD turbulence, we first need to relate $Q$ to the wave vector anisotropy in terms of turbulence theories. These are usually based on the analysis of the structure of the eddies as a function of scale in presence of a local magnetic field. Parallel and perpendicular to the magnetic field eddies become correlated. As the turbulent cascade proceeds to larger spatial frequencies the eddies become more and more elongated along the direction of the magnetic field. The deformation increases until dissipation is affecting the turbulent flow (Goldreich \& Sridhar 1995) for recent reviews see Cho et al. (2003), Brandenburg \& Lazarian (2013).

For a description how eddies (or wave packets) are related to the corresponding wave vectors see Cho \& Vishniac (2000, Fig. 6). For a wave packet with extension $L_{1}$ perpendicular to the local magnetic field direction and $L_{2}$ parallel to the field, the corresponding wave vectors in Fourier space are for $L_{1}$ transformed to $1 / k_{\perp}$ and for $L_{2}$ respectively to $1 / k_{\|}$. Anisotropy implies in the Fourier domain isophotes with scales $k_{\|}<k_{\perp}$. The power spectra $P_{\|}$and $P_{\perp}$ are defined correspondingly, $P_{\|}(k)<P_{\perp}(k)$.

According to Goldreich \& Sridhar (1995), turbulence spectra $P_{\perp}$ are in Alfvén and slow modes nearly unaffected by the magnetic field with power spectra close to the isotropic case.

\subsection{Field direction and spectral index}

We determined spectral indices $\gamma$ with respect to the field angle $\Delta \Phi=\Phi-\Phi_{\perp}$, restricting the fit to spatial frequencies with clear anisotropies, $0.005<k<0.05$. Figure 23 shows the results for two cases, the channel at $v_{\mathrm{LSR}}=-3.76 \mathrm{~km} \mathrm{~s}^{-1}$ (black) and the velocity centroid (red). We find in both cases a complex distribution but a general trend for a steep power index in field direction, consistent with the mean (isotropical) spectral indices indicated with the horizontal lines in Fig. 23. Parallel to the magnetic field, for $\Delta \Phi \sim 90^{\circ}$, the spectral indices flatten, approaching $\gamma \sim-2.1$. Goldreich \& Sridhar (1995) predict for $P_{\perp}$ that the spectral index for anisotropic turbulence is nearly identical to the isotropic case as indicated by the horizontal lines in Fig. 23. This prediction is in good agreement with our result.

\subsection{Anisotropies $Q\left(v_{\mathrm{LSR}}, k\right)$}

From observations, $Q_{\text {aver }}$ appears far more useful to characterize anisotropic turbulence than fitting spectral indices to power 
3C 196 field, spectral index distribution for $0.005<\mathrm{k}<0.05$

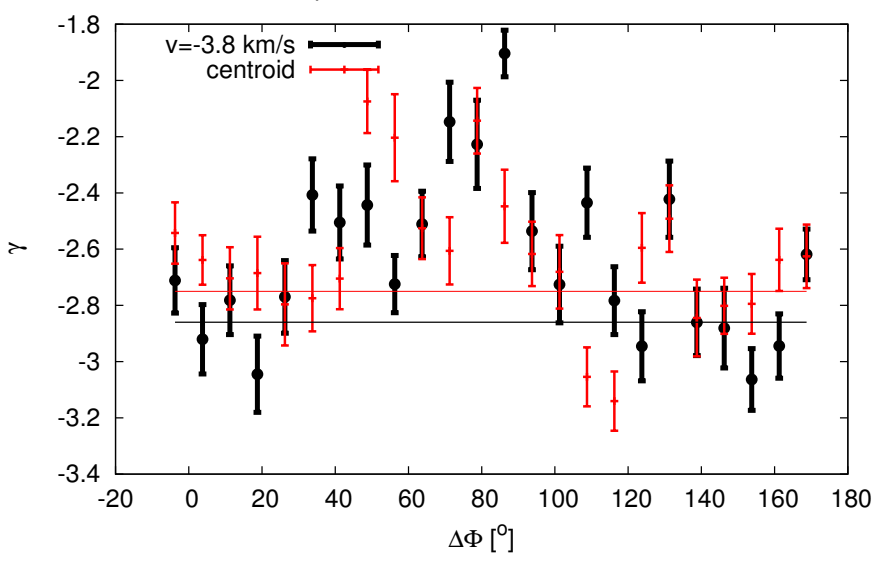

Fig. 23. Distribution of spectral index $\gamma$ with respect to the field angle $\Delta \Phi=\Phi-\Phi_{\perp}$. Fits for individual sectors with width $2 \delta \Phi=7.5$ (black) are shown for the channels at $v_{\mathrm{LSR}}=-3.76 \mathrm{~km} \mathrm{~s}^{-1}$ and the velocity centroid (red). Only spatial frequencies $0.005<k<0.05 \mathrm{arcmin}^{-1}$ are considered. Isotropic spectral indices are plotted with horizontal lines for comparison.

spectra. Here we try to go a step further to find out whether $Q(k)$ can be used to determine systematic changes in spectral indices.

Figure 8 shows that anisotropies have a constant position angle for velocities $-11.5<v_{\mathrm{LSR}}<-1.2 \mathrm{~km} \mathrm{~s}^{-1}$, also $Q_{\text {aver }}$ is well defined in this range (Fig. 12). We choose spectral channels that may be considered as independent and typical for this range. In Fig. 24 we display $Q(k)$ for the channel map at $v_{\mathrm{LSR}}=-6.3 \mathrm{~km} \mathrm{~s}^{-1}$ (black dots), $v_{\mathrm{LSR}}=-8.9 \mathrm{~km} \mathrm{~s}^{-1}$ (blue), and for the velocity centroid (red). There is quite some scatter but we obtain a general increase of the anisotropies up to $k \sim 0.025 \mathrm{arcmin}^{-1}$. For $0.025 \lesssim k \lesssim 0.07 \operatorname{arcmin}^{-1}$ (enclosed by horizontal lines) the anisotropies appear to decay, the highest spatial frequencies are noise dominated and cannot be interpreted. For the rising branch we fit for each of our samples a power law $Q(k) \propto k^{\beta}$ and obtain $0.59 \lesssim \beta \lesssim 0.81$. We find clear evidence that anisotropies increase with spatial frequencies as predicted by Goldreich \& Sridhar (1995) but the slope appears not to be very well defined. The observed data are affected by local effects that cause quite some scatter.

\subsection{Decay of anisotropies?}

For $k>0.025 \operatorname{arcmin}^{-1}$ we find in Fig. 24 a general systematical decrease of $Q(k)$. This tail suggests a decay of the observed anisotropies in the same sense as the energy decays in the turbulent flow to progressively smaller scales. The question arises whether this apparent decay may be caused by instrumental limitations. A decay may be mimicked by noise limitations. We calculate the signal-to-noise ratio $(\mathrm{S} / \mathrm{N})$ of the anisotropies by comparing $P_{\|}(k)$ with the power in the matched noise template $N(k)$ (compare Fig. 9 with Fig. 6). For $k<0.054 \operatorname{arcmin}^{-1}$ we find a $\mathrm{S} / \mathrm{N}$ of three or better. The $\mathrm{S} / \mathrm{N}$ for $P_{\perp}(k=0.054)$ is more than five times better. Hence $Q(k)$ is for $k<0.054 \mathrm{arcmin}^{-1}$ significant and unaffected by instrumental problems. Numerical uncertainties may also cause limitations. We use the Singleton FFT algorithm in double precision and are not aware about accuracy limitations.

Common in Fig. 24 is that the increase of the anisotropies $Q(k)$ is accompanied with quite some scatter. This scatter is much stronger then the expected random noise and appears to indicate local but systematical deviations caused by external anisotropies in the 3C 196 field, fits for $0.0015<\mathrm{k}<0.025 \mathrm{arcmin}^{-1}$

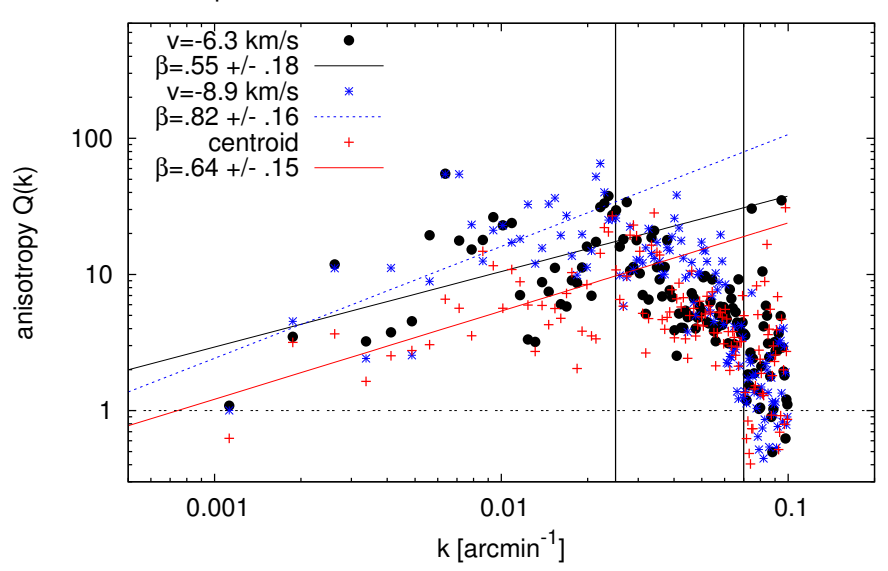

Fig. 24. Anisotropies $Q(k)$ for the channels at $v_{\mathrm{LSR}}=-6.3$, $-8.9 \mathrm{~km} \mathrm{~s}^{-1}$ (black and blue), and for the velocity centroid (red). Power laws $Q \propto k^{\beta}$ were fit for $k<0.025 \operatorname{arcmin}^{-1}$.

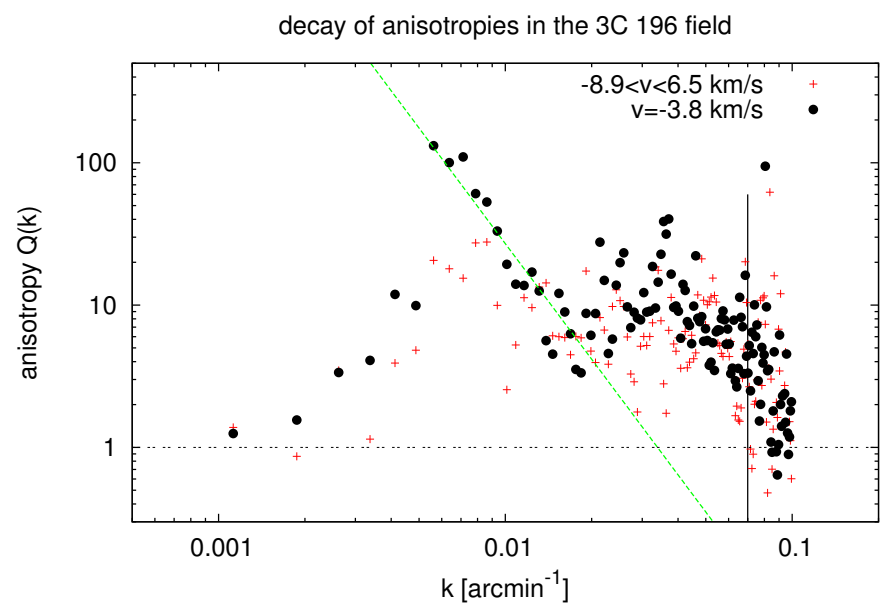

Fig. 25. Anisotropies $Q(k)$ for the channel at $v_{\mathrm{LSR}}=-3.76 \mathrm{~km} \mathrm{~s}^{-1}$ (black dots). The strong local anisotropy of $Q \sim 130$ at $k=0.0056 \mathrm{arcmin}^{-1}$ decays as $Q \propto k^{-2.7}$. Also the anisotropies at $k>0.04 \operatorname{arcmin}^{-1}$ appear to decay in a similar way. For comparison we show thick slice anisotropies for $-8.9<v_{\mathrm{LSR}}<6.5 \mathrm{~km} \mathrm{~s}^{-1}$ with red crosses.

forces. The channel map at $v_{\mathrm{LSR}}=-3.76 \mathrm{~km} \mathrm{~s}^{-1}$, shown in Fig. 25, has the strongest local scatter with the strongest anisotropy of $Q \sim 130$ at $k=0.0056 \operatorname{arcmin}^{-1}$. Considering the turbulent flow from large eddies to smaller ones, this outstanding anisotropy pops suddenly up (by a factor of ten), is highly significant but inconsistent with the typical anisotropies that are observed at other velocities. Towards high spatial frequencies this anisotropy decays as $Q \propto k^{-2.70 \pm .24}$ (green line in Fig. 25), a slope that is in good agreement with the 2D Kolmogorov index.

It may be fortuitous to relate the decaying part of the anisotropies to the Kolmogorov index. Figure 26 shows that the strongest anisotropy in the FN1 field has a comparable pattern with a rising part at low spatial frequencies up to $Q=74$ at $k=0.0064$. This branch can be fit with a power law index $\gamma=2.4 \pm 0.4$. The anisotropies appear to decay for larger spatial frequencies with a power law index $\gamma=-1.6 \pm 0.1$. These spectral indices differ significantly from the results obtained for 3C 196, possibly indicating different processes causing the anisotropies. The 3C 196 filaments are clearly aligned with the magnetic field but using $353 \mathrm{GHz}$ Planck data for the FN1 field it is hardly possible to relate the filament to a magnetic field direction. The average magnetic field position angle for an area with 


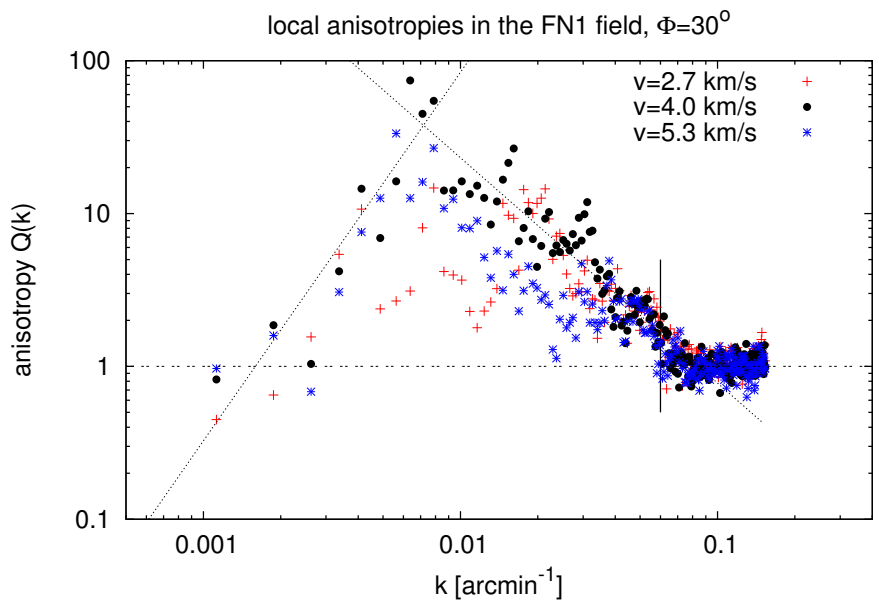

Fig. 26. Anisotropies $Q(k)$ in the FN1 field for the channel at $v_{\mathrm{LSR}}=$ $4.0 \mathrm{~km} \mathrm{~s}^{-1}$ (black dots) and neighbor channels at $v_{\mathrm{LSR}}=2.7 \mathrm{~km} \mathrm{~s}^{-1}$ (red) and $v_{\mathrm{LSR}}=5.3 \mathrm{~km} \mathrm{~s}^{-1}$ (blue). Anisotropies at $v_{\mathrm{LSR}}=4.0 \mathrm{~km} \mathrm{~s}^{-1}$ appear to rise with a spectral index of $\gamma \sim 2.4$ and decay with $\gamma \sim$ -1.6 (dotted lines).

a diamenter of $10^{\circ}$ is $\phi_{\mathrm{mag}}=111^{\circ} .4 \pm 2.2$ and $\phi_{\mathrm{mag}}=106.4 \pm 1.0$ respectively for a diamenter of $20^{\circ}$ (F. Boulanger, private communication). Different processes may cause different spectral indices.

The strong FN1 anisotropy is caused by CNM with a geometric mean Doppler temperature of $T_{\mathrm{D}} \sim 250 \mathrm{~K}$. Due to the low temperature, neighbor channels are nearly uncorrelated and show significantly different anisotropies. The position angles show significant changes (Fig. 17), it is not possible to assign an unambiguous relation between filament and magnetic field direction. We found peak anisotropies $\phi \sim 30^{\circ}$ for $v_{\mathrm{LSR}}=$ $3.97 \mathrm{~km} \mathrm{~s}^{-1}$. The velocity channel at $v_{\mathrm{LSR}}=10.4 \mathrm{~km} \mathrm{~s}^{-1}$ has $\phi=9.3^{\circ} \pm 13^{\circ}$ with a peak anisotropy of $Q=55$, the channel at $v_{\mathrm{LSR}}=-1.2 \mathrm{~km} \mathrm{~s}^{-1}$ has $\phi=40^{\circ} \pm 11^{\circ}$ with a peak anisotropy of $Q=10$ respectively. In both cases we derive Doppler temperatures $T_{\mathrm{D}} \lesssim 350 \mathrm{~K}$, the HI gas belongs to the CNM. Miville-Deschênes \& Martin (2007) argue that 2/3 of the column density in the FN1 region is made of WNM and $1 / 3$ of thermally unstable gas $(T \sim 2600 \mathrm{~K})$. They further find that this field contains a signature of CNM type gas at a very low level which could have been formed by a convergent flow of WNM gas. Details of the CNM component are not specified but we conclude that this gas probably corresponds to the component that shows anisotropies.

The 3C 196 filament showing strong anisotropies at $v_{\mathrm{LSR}}=$ $-3.76 \mathrm{~km} \mathrm{~s}^{-1}$ is aligned with the magnetic field and is of particular interest since the CNM HI distribution at this velocity shows the best visible correlation of all HI USM channel maps with the LOFAR filaments, see Fig. 2. The strong local anisotropy is also visible in Fig. 9. Perpendicular to the magnetic field (red) the spectral power increases locally with $k$ while there is a stagnation in parallel direction (green). We interpret this as an indication for a strong local interaction.

Assuming that the observed anisotropies are caused by MHD turbulence, we might consider the case whether the magnetic field could cease at $k \gtrsim 0.025 \mathrm{arcmin}^{-1}$. It is difficult to prevent the magnetic field from small scales but from the deformation of eddy shapes, reconnection appears to be a possibility. Lazarian \& Vishniac (1999, Eq. (A.13)) estimated for this case under typical CNM conditions a scale of 0.03 pc. For dissipation effects, the only known mechanism in the ISM that leads to comparable scales is the ion-neutral friction. Hennebelle (2013) estimate the length associated to the ion-neutral friction in the typical $\mathrm{CNM}$ to be in the range $3 \times 10^{-3}$ to $2 \times 10^{-2} \mathrm{pc}$. We may compare these scales with estimates of the filament thickness from observations. For unresolved filaments, assumed to be sheets seen edge-on, Kalberla et al. (2016) estimate a median thickness of $0.3 \mathrm{pc}$, and in case that magnetic pressure confinement applies, a thickness of $0.1 \mathrm{pc}$. McClure-Griffiths et al. (2006) and Clark et al. (2014) obtained comparable results.

For local HI filaments at a distance of $100 \mathrm{pc}$, based on the median distance to the wall of the local cavity (Lallement et al. 2014, from color excess measurements), the break in the anisotropy distribution at $k \sim 0.025 \mathrm{arcmin}^{-1}$ corresponds to an eddy extension of $l \sim 1.2 \mathrm{pc}$ perpendicular to the magnetic field. This is by far too large to explain a decay of anisotropies by reconnection or dissipation by ion-neutral friction. A different explanation needs to be found.

\subsection{Spectral index dependencies}

We find in case of well defined anisotropies in the range $0.005<$ $k<0.05 \operatorname{arcmin}^{-1}$ always $Q(k)=P_{\perp}(k) / P_{\|}(k) \gg 1$. The spectral index $\gamma_{\perp}$ for $P_{\perp}$ is well defined and close to the isotropic index $\gamma_{\text {iso }} \sim-8 / 3$, one of the basic predictions by Goldreich \& Sridhar (1995). For $P_{\|}(k)$ we obtain an average power index $\gamma_{\|}=$ $-2.06 \pm 0.2$ (Fig. 23).

According to $Q(k)=P_{\perp}(k) / P_{\|}(k) \propto k^{\gamma_{\perp}} / k^{\gamma_{\|}}=k^{\beta}$ this implies that anisotropies increase with a spectral index $\beta \sim 2 / 3$, we fit $0.59<\beta<0.81$ (see Fig. 24), consistent with the second prediction by Goldreich \& Sridhar (1995).

However, anisotropies do not increase in an unlimited way. Our data suggest that anistropies decrease for $k \gtrsim$ $0.025 \mathrm{arcmin}^{-1}$. Also the power distribution for the channel at $v_{\text {LSR }}=-3.76 \mathrm{~km} \mathrm{~s}^{-1}$ does not agree with the predictions. At $k=$ $0.0056 \operatorname{arcmin}^{-1}$ we find a strong anisotropy $Q(k) \sim 130$ which is inconsistent with MHD model assumptions. This anisotropy decays according to $Q(k)=P_{\perp}(k) / P_{\|}(k) \propto k^{\gamma_{\perp}} / k^{\gamma_{\|}}=k^{\beta} \sim k^{-2.7}$. For this channel we measure $\gamma_{\text {iso }} \sim \gamma_{\perp} \sim-2.86$, implying $\gamma_{\|} \sim 0.16$. Within the errors the power distribution parallel to the field is flat, $P_{\|}=$const. The flat part of the power distribution can be seen in Fig. 9 (green) for $0.0056<k<0.02 \mathrm{arcmin}^{-1}$. Quite surprisingly, we observe for the affected range in spatial frequencies a signal that is completely uncorrelated. For $P_{\|}(k)$ the turbulent cascade is suspended, there is no turbulent energy transport from larger to smaller eddies. This interpretation assumes that the $P_{\perp}$ distribution is largely unaffected by the decay and close to $P_{\text {iso }}$ as predicted by Goldreich \& Sridhar (1995).

\subsection{Eddy shapes}

For local Hi filaments we estimate a distance of 100 pc, based on Lallement et al. (2014). The filaments extend approximately over $20^{\circ}$ (see Fig. 1), corresponding to an outer turbulence scale of $l_{\mathrm{o}} \sim 35 \mathrm{pc}$. The break in the anisotropy distribution at $k \sim$ $0.025 \mathrm{arcmin}^{-1}$ corresponds for this distance to an average eddy extension of $40^{\prime}$ or $l \sim 1.2 \mathrm{pc}$ perpendicular to the magnetic field. For a power law index of $\gamma=-8 / 3$ the wave vector anisotropy is $k_{\perp} / k_{\|} \sim Q^{3 / 8} \sim 2.4$ (Chen et al. 2010). Hence the dominant eddy size is $1.2 \mathrm{pc}$ by $2.8 \mathrm{pc}$. For $Q=130$ we get accordingly an eddy shape that is typically 6.2 times more extended along the field line than perpendicular. The size is here $5.2 \mathrm{pc}$ by $32 \mathrm{pc}$. In Fig. 27 we display eddy anisotropies $L_{\|} / L_{\perp}$ as function of spatial frequency. 


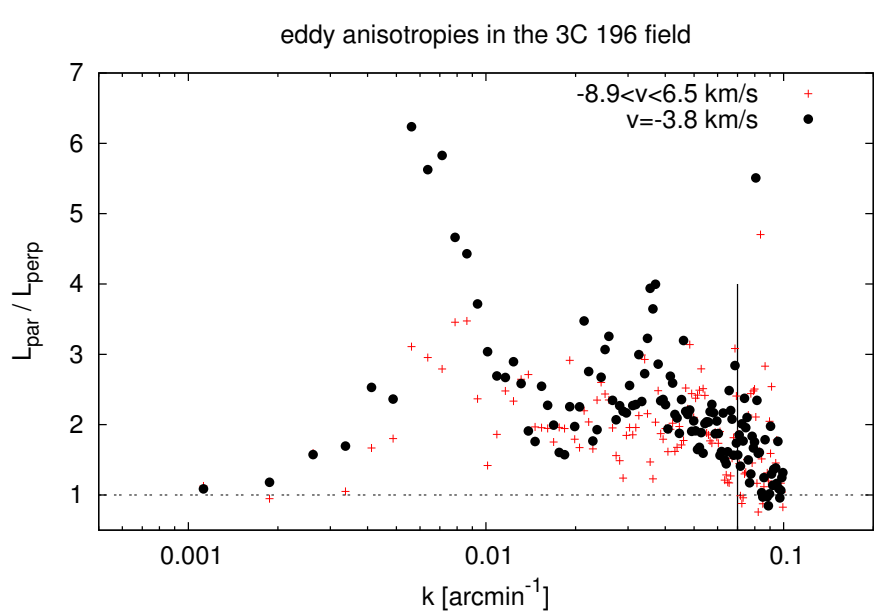

Fig. 27. Eddy anisotropies $L_{\|} / L_{\perp} \propto Q(k)^{3 / 8}$ for the channel at $v_{\mathrm{LSR}}=$ $-3.76 \mathrm{~km} \mathrm{~s}^{-1}$ (black dots). Thick slice eddy anisotropies for $-8.9<$ $v_{\mathrm{LSR}}<6.5 \mathrm{~km} \mathrm{~s}^{-1}$ are added for comparison (red crosses).

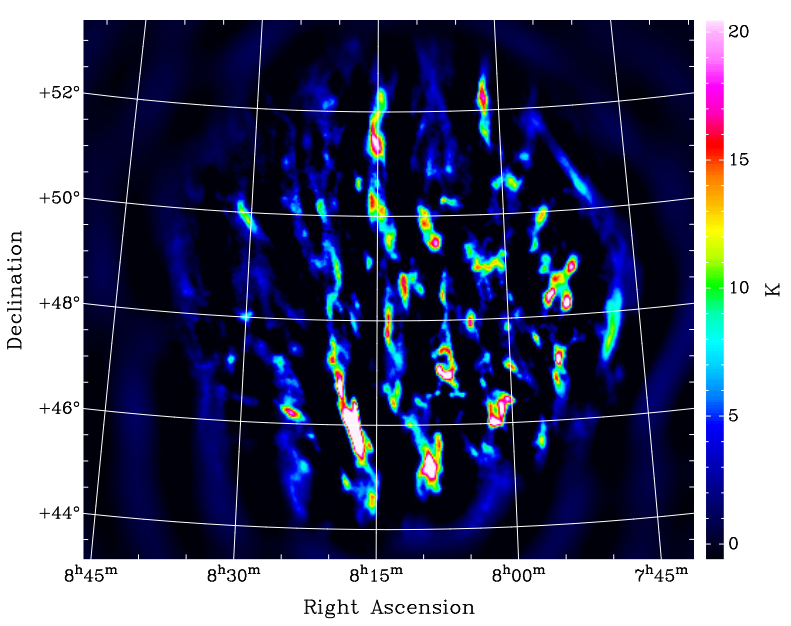

Fig. 28. Apodized brightness temperature distribution in direction to $3 \mathrm{C} 196$ at $v_{\mathrm{LSR}}=-3.76 \mathrm{~km} \mathrm{~s}^{-1}$ for spatial frequencies $k>$ $0.0056 \operatorname{arcmin}^{-1}$ with anisotropies $Q<130$.

In Fig. 28 we display data for the channel at $v_{\mathrm{LSR}}=$ $-3.76 \mathrm{~km} \mathrm{~s}^{-1}$ for spatial frequencies $k>0.0056 \mathrm{arcmin}^{-1}$. From our data this is the most extreme anisotropy and probably the best possible visualization of what one may consider as eddies or wave packets. For comparison we plot in Fig. 29 the CNM gas, indicating that these eddies coincide with rather cold HI gas. In this case we derive the average anisotropies $Q_{\text {aver }}=11$ but the peak anisotropy reaches $Q=65$, only $50 \%$ compared to the HI channel map in Fig. 28. When generating USM maps, spatial frequencies $k \lesssim 0.033 \operatorname{arcmin}^{-1}$ are attenuated including the anisotropies observed at $0.01<k<0.03 \mathrm{arcmin}^{-1}$. Due to the fact that MHD driven anisotropies increase with spatial frequency, USM maps give a good first order approximation of the anisotropic distribution of the HI at high spatial frequencies. The visual inspection of the USM map implies that most of the filaments are unresolved.

\section{Summary and conclusions}

It was recently shown that a major part of the $\mathrm{CNM}$ is organized in cold filaments with median Doppler temperatures $T_{\mathrm{D}} \sim 223 \mathrm{~K}$ that are aligned with the magnetic field (Kalberla et al. 2016).

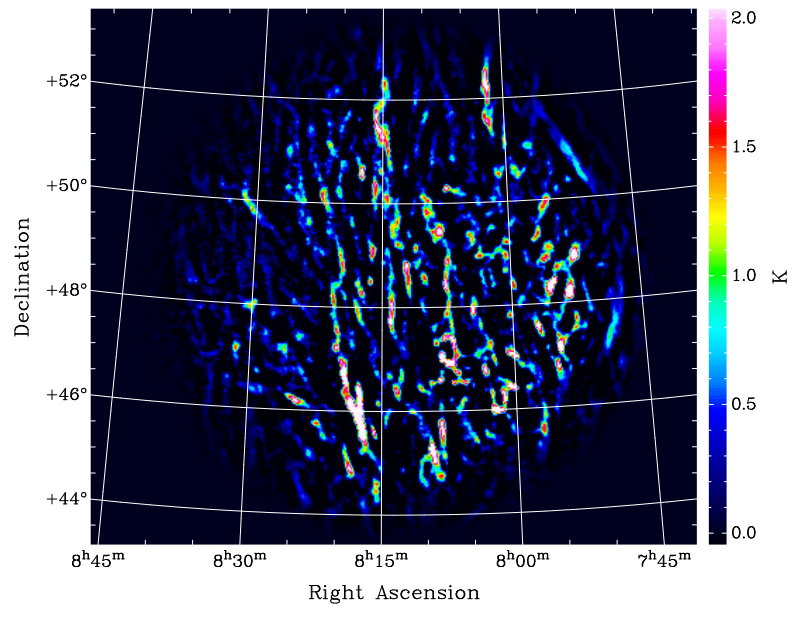

Fig. 29. Apodized USM temperature map for the CNM in direction to 3C 196 at $v_{\text {LSR }}=-3.76 \mathrm{~km} \mathrm{~s}^{-1}$, demonstrating that the CNM is dominated by filaments. Anisotropies at high spatial frequencies can be inferred from CNM maps.

These structures can be highly anisotropic with aspect ratios up to 400 (Heiles \& Troland 2005; McClure-Griffiths et al. 2006; Clark et al. 2014; Kalberla et al. 2016). Such a medium is expected to be driven by MHD turbulence and asymmetries should have a large impact on the dynamics of the turbulent cascade.

To determine anisotropies in MHD driven turbulence we decided to analyze the HI distribution in direction to 3C 196. This field is one of the primary fields of the LOFAR-Epoch of Reionization key science project. Linear polarization structures have been observed with LOFAR by Jelić et al. (2015). Strikingly straight filaments were found to be aligned with the magnetic field (Zaroubi et al. 2015) and HI shows a similar filamentary structure (Fig. 2).

We find that average power spectra, as well as power spectra perpendicular to the magnetic field, are for thin slices in velocity close to the expected Kolmogorov index $\gamma=-8 / 3$. The general impression is that in case of anisotropies the parallel and perpendicular power spectra appear to the first order in logarithmic presentation just "offset" from the isotropic distribution, see Figs. 7 to 11, 14, and 15. More important are however local and correlated deviations from the isotropic power distribution. The data show quite some fluctuations which we interpret as caused by external forces.

The HI distribution in direction to 3C 196 has two major components (Fig. 12), at $v_{\mathrm{LSR}} \sim-1 \mathrm{~km} \mathrm{~s}^{-1}$ and $\sim 13 \mathrm{~km} \mathrm{~s}^{-1}$ but only the component at $v_{\mathrm{LSR}} \sim-1 \mathrm{~km} \mathrm{~s}^{-1}$ appears visually to be related to the LOFAR filaments. We observe strong average anisotropies, $10<Q_{\text {aver }}<20$, in the spectral power distribution of the $v_{\mathrm{LSR}} \sim-1 \mathrm{~km} \mathrm{~s}^{-1}$ component. The $v_{\mathrm{LSR}} \sim$ $13 \mathrm{~km} \mathrm{~s}^{-1}$ component does not show significant anisotropies. Thus anisotropies are clearly linked to HI filaments that appear visually to be correlated with LOFAR filaments. The analysis of two more comparison fields show that anisotropies, unrelated to filamentary structures, are mostly restricted to $Q_{\text {aver }} \sim 2$.

The derived anisotropy position angle (Fig. 7) agrees well with the orientation of the LOFAR filaments (Jelić et al. 2015) and the direction of the associated magnetic field, derived from Planck $353 \mathrm{GHz}$ data (Zaroubi et al. 2015). The anisotropies are caused by cold CNM filaments, we derive a geometric mean Doppler temperature $T_{\mathrm{D}} \sim 161 \mathrm{~K}$. The power law index $\gamma$ tends 
to be steepest close to the minima of the Doppler temperature. Anisotropies $Q_{\text {aver }}$ are not correlated with Doppler temperature or average line emission, also we found no correlation with the power law index $\gamma$. Anisotropies lead to significant changes in the power distribution, $P_{\|}(k)<P_{\perp}(k)$. Typical ratios reach $Q(k)=P_{\perp}(k) / P_{\|}(k) \sim 10$ to 20 , peaking at $Q(k) \sim 130$. Anisotropies increase with spatial frequency, $Q(k) \propto k^{\beta}$ with $0.59 \lesssim \beta \lesssim 0.81$ until $k \sim 0.025 \operatorname{arcmin}^{-1}$.

We observe a decay of anisotropies at high spatial frequencies $k \gtrsim 0.025 \operatorname{arcmin}^{-1}$. From theory, MHD anisotropies should increase with spatial frequency until a scale is reached where the turbulent flow becomes dissipative. However we find no indications for a turnover in the isotropic spectral distribution that might be considered as an indication for dissipation. The average power distribution remains unchanged. All we notice is a systematic decrease of the anisotropies. Also the angular power spectrum of integrated polarized emission, derived by Jelić et al. (2015) from LOFAR data, shows no change in spectral slope down to the resolution limit at a scale of 4 armin.

A similar anisotropy decay with a spectral index of $\gamma \sim-2.7$ is observed for the extreme case $Q \sim 130$ in the 3C 196 field. While the power law index $\gamma_{\perp}(k)$ is almost unaffected by the decay we find here within the errors $\gamma_{\|}(k) \sim 0$, implying for the decay branch that there is no turbulent energy transport for $P_{\|}(k)$. The turbulent cascade appears to be suspended for $P_{\|}(k)$ until $k \sim 0.02 \mathrm{arcmin}^{-1}$ with an anisotropy of $4 \lesssim Q \lesssim 8$, typical for the other velocity channels, is reached. In other words, this process is terminated when anisotropy according to the relation $Q(k) \propto k^{2 / 3}$ (Goldreich \& Sridhar 1995) is reached.

Our interpretation for the $Q \sim 130$ case is that for the scale length (eddy size) under consideration the enhanced power $P_{\perp}(k)$ was forced by an event that affected the eddy shape in a sense that power from $P_{\|}(k)$ was transferred to $P_{\perp}(k)$ (compression of the eddy). After the external force is removed, the turbulent cascade continues for a while in an isotropic way; the eddy shapes need to relax according to the $Q(k) \propto k^{2 / 3}$ condition.

Our interpretation of the 3C 196 anisotropies may be fortuitous, the processes causing these anisotropies are not yet understood. In case of our comparison field, the FN1 region, we observe for a narrow velocity interval another strong anisotropy (peak at $Q=74$ ) but with significant different spectral indices concerning the rise and decay of anisotropies with spatial frequency (Fig. 26).

In our analysis we compare power spectra derived from thin and thick slices as well as from centroid maps. The predicted steepening of power indices (Lazarian \& Pogosyan 2000), comparing thin and thick slices, is only partly verified by us. We find that anisotropies are always best defined for thin slices. Figure 25 demonstrates that thick slices show less anisotropies and a further comparison with Fig. 24 leads to the result that velocity centroids, calculated for the same velocity range, may even be less suitable to characterize anisotropies. Interestingly, our result contradicts one of the main results by Kandel et al. (2016), who finds that turbulence anisotropies increase with velocity slice thickness.

The interpretation of our result is straightforward. Since the filamentary CNM in the 3C 196 field is very cold $\left(T_{\mathrm{D}} \sim 160 \mathrm{~K}\right)$, adjacent velocity channels are uncorrelated for $\delta v_{\mathrm{LSR}} \gtrsim 3 \mathrm{~km} \mathrm{~s}^{-1}$. On the other hand filaments in neighboring channels show very similar structures. Filaments are probably HI sheets seen edgeon. Different slices, systematically offset from each other, are observed at different velocities. These structures are not random but correlated. Integrating the data to obtain thick slices or velocity centroids means for the analysis that local (uncorrelated) anisotropies are smoothed out. Filaments, running parallel (see Figs. 10, and 25 to 27 of Kalberla et al. 2016), are broadened artificially by averaging and anisotropy is lost. The fluctuations caused by the CNM within a single thin velocity slice is dominated by density effects but changes in the observed density distribution with velocity are correlated because of global anisotropies, probably influenced by the magnetic field, that extend over a few channels.

For the FN1 field we find that position angles change systematically with velocity. The CNM that is associated with the anisotropies is also in this case sufficiently cold that neighbor channels are nearly independent. Calculating the mean emission or the velocity centroid over a range of velocities (in a thick slice), anisotropies smooth out and loose significance. The result from our investigations is clearly that anisotropies are associated with the CNM and not with the more extended but diffuse WNM.

Alfvén modes are incompressible and to the first order approximation they do not create any density fluctuations. The expected power law index is $\gamma=-11 / 3$ (Kandel et al. 2016). In Sect. 4.2 we excluded fast modes since these would cause eddies elongated perpendicular to the magnetic field. The power spectrum in this case would be isotropic with an index of $\gamma=-9 / 2$. For both modes this is not what we observe. We conclude that we probably observe slow modes in a particular favorable condition, the magnetic field is mostly oriented perpendicular to the line of sight. According to (Heiles \& Troland 2005) the expected average field strength is $6 \mu \mathrm{G}$, the turbulent $\beta \sim 0.3$, the Alfvénic Mach number $M_{\mathrm{A}} \sim 1.1$, and the Alfvén velocity $v_{\mathrm{A}} \sim 1.5 \mathrm{~km} \mathrm{~s}^{-1}$. LOFAR observations and polarized dust emission by Planck restrict the total magnetic field strength for the central filament to $B_{\text {tot }} \lesssim 6.5 \mu \mathrm{G}$ and the component along the line of sight to $B \gtrsim 1.2 \mu \mathrm{G}$. The pulsar J081558+461155, is located about $2^{\circ}$ south of 3C 196. Jelić et al. (2015) derived from the RM to dispersion measure ratio a mean line-of-sight magnetic field component of $B_{\|}=0.3 \pm 0.1 \mu \mathrm{G}$ and its variations across the 3C 196 field to $0.1 \mu \mathrm{G}$. The 3C 196 field is well studied and we hope that follow-up investigations can lead to more precise results.

The morphology of HI filaments in the 3C 196 field is typical for the CNM studied by Kalberla et al. (2016). For "unperturbed" HI , thus for regions that are not affected by obvious CNM filaments, we find only weak anisotropies around $Q_{\text {aver }} \sim 2$. But this result, as well as examples and results for strong anisotropies are based on a rather limited sample.

This is to our knowledge the first study of turbulent anisotropies in the HI gas. Some more investigations are necessary for a generalization of our results, but the EBHIS (Winkel et al. 2016a,b), as well as the GASS III (Kalberla \& Haud 2015), are available for such an analysis. The only limitation of these surveys is caused by the different beam sizes, because of different telescope diameters. That disables an analysis of fields close to the ecliptic equator. The LDS, as well as the Leiden/Argentine/Bonn survey (LAB, Kalberla et al. 2005) are obsolete and should no longer be used to determine properties of a turbulent flow. Our results are incompatible with previous investigations using the LDS.

Acknowledgements. We thank F. Boulanger for providing data on the magnetic field direction in the FN1 region and V. Jelić for comments. The authors thank the Deutsche Forschungsgemeinschaft (DFG) for support under grant numbers KE757/7-1, KE757/7-2, KE757/7-3 and KE757/11-1. This research has made use of NASA's Astrophysics Data System. EBHIS is based on observations with the 100-m telescope of the MPIfR (Max-Planck-Institut für Radioastronomie) at Effelsberg. 
P. M. W. Kalberla and J. Kerp: HI anisotropies

\section{References}

Blagrave, K., Martin, P. G., Joncas, G., et al. 2016, ApJ, submitted [arXiv: 1602.02115]

Bracewell, R. N. 2000, The Fourier transform and its applications, ed. R. N. Bracewell (Boston: McGraw Hill)

Brandenburg, A., \& Lazarian, A. 2013, Space Sci. Rev., 178, 163

Briggs D., 1995, Ph.D. Thesis, New Mexico Institude of Mining and Technology

Chen, C. H. K., Wicks, R. T., Horbury, T. S., \& Schekochihin, A. A. 2010, ApJ, 711, L79

Cho, J., Lazarian, A., \& Vishniac, E. T. 2003, in Turbulence and Magnetic Fields in Astrophysics, Lect. Notes Phys., 614, 56

Cho, J., \& Vishniac, E. T. 2000, ApJ, 539, 273

Clark, S. E., Peek, J. E. G., \& Putman, M. E. 2014, ApJ, 789, 82

Crovisier, J., \& Dickey, J. M. 1983, A\&A, 122, 282

Crovisier, J., Kazes, I., \& Aubry, D. 1978, A\&AS, 32, 205

Dedes, L., \& Kalberla, P. M. W. 2012, EAS Publ. Ser., 56, 209

Dickey, J. M., McClure-Griffiths, N. M., Stanimirović, S., Gaensler, B. M., \& Green, A. J. 2001, ApJ, 561, 264

Dole, H., Gispert, R., Lagache, G., et al. 2001, A\&A, 372, 364

Esquivel, A., \& Lazarian, A. 2005, ApJ, 631, 320

Goldreich, P., \& Sridhar, S. 1995, ApJ, 438, 763

Green, D. A. 1993, MNRAS, 262, 327

Harris, F. J. 1978, IEEE Proc., 66, 51

Hartmann, D., \& Burton, W. B. 1997, in Atlas of Galactic Neutral Hydrogen, eds. D. Hartmann, \& W. B. Burton (Cambridge, UK: Cambridge University Press), 243

Heiles, C., \& Troland, T. H. 2003, ApJ, 586, 1067

Heiles, C., \& Troland, T. H. 2005, ApJ, 624, 773

Hennebelle, P. 2013, A\&A, 556, A153

Jelić, V., de Bruyn, A. G., Pandey, V. N., et al. 2015, A\&A, 583, A137
Kalberla, P. M. W., \& Haud, U. 2015, A\&A, 578, A78

Kalberla, P. M. W., \& Mebold, U. 1983, Proc. of Conf., Mitteilungen der Astronomischen Gesellschaft Hamburg, 58, 101

Kalberla, P. M. W., Burton, W. B., Hartmann, D. et al. 2005, A\&A, 440, 775

Kalberla, P. M. W., McClure-Griffiths, N. M., Pisano, D. J., et al. 2010, A\&A, 521, A17

Kalberla, P. M. W., Kerp, J., Haud, U., et al. 2016, ApJ, 821, 117

Kandel, D., Lazarian, A., \& Pogosyan, D. 2016, MNRAS, 461, 1227

Lallement, R., Vergely, J.-L., Valette, B., et al. 2014, A\&A, 561, A91

Lazarian, A., \& Pogosyan, D. 2000, ApJ, 537, 720

Lazarian, A., \& Vishniac, E. T. 1999, ApJ, 517, 700

McClure-Griffiths, N. M., Dickey, J. M., Gaensler, B. M., Green, A. J., \& Haverkorn, M. 2006, ApJ, 652, 1339

McClure-Griffiths, N. M., Pisano, D. J., Calabretta, M. R., et al. 2009, ApJS, 181,398

Martin, P. G., Blagrave, K. P. M., Lockman, F. J., et al. 2015, ApJ, 809, 153

Miville-Deschênes, M.-A., \& Martin, P. G. 2007, A\&A, 469, 189

Miville-Deschênes, M.-A., Joncas, G., Falgarone, E., \& Boulanger, F. 2003, A\&A, 411, 109

Mebold, U., Winnberg, A., Kalberla, P. M. W., \& Goss, W. M. 1982, A\&A, 115, 223

Planck Collaboration XXXII. 2016, A\&A, 586, A135

Saury, E., Miville-Deschênes, M.-A., Hennebelle, P., Audit, E., \& Schmidt, W. 2014, A\&A, 567, A16

Stanimirović, S., \& Lazarian, A. 2001, ApJ, 551, L53

Stanimirović, S., Staveley-Smith, L., Dickey, J. M., Sault, R. J., \& Snowden, S. L. 1999, MNRAS, 302, 417

von Weizsäcker, C. F. 1951, ApJ, 114, 165

Winkel, B., Kerp, J., Flöer, L., et al. 2016a, A\&A, 585, A41

Winkel, B., Lenz, D., \& Flöer, L. 2016b, A\&A, 591, A12

Zaroubi, S., Jelić, V., de Bruyn, A. G., et al. 2015, MNRAS, 454, L46 